\title{
On the Convergence to Equilibrium of Unbounded Observables Under a Family of Intermittent Interval Maps
}

\author{
Johannes Kautzsch, Marc Kesseböhmer and Tony Samuel
}

\begin{abstract}
We consider a family $\left\{T_{r}:[0,1] \circlearrowleft\right\}_{r \in[0,1]}$ of Markov interval maps interpolating between the tent map $T_{0}$ and the Farey map $T_{1}$. Letting $\mathcal{P}_{r}$ denote the Perron-Frobenius operator of $T_{r}$, we show, for $\beta \in[0,1]$ and $\alpha \in(0,1)$, that the asymptotic behaviour of the iterates of $\mathcal{P}_{r}$ applied to observables with a singularity at $\beta$ of order $\alpha$ is dependent on the structure of the $\omega$-limit set of $\beta$ with respect to $T_{r}$. The results presented here are some of the first to deal with convergence to equilibrium of observables with singularities.
\end{abstract}

\section{Introduction}

Expanding maps of the unit interval have been widely studied in the last decades and the associated transfer operators have proven to be of vital importance in solving problems concerning the statistical behaviour of the underlying interval maps $[3,5,34]$.

In recent years, an increasing amount of interest has developed in maps which are expanding everywhere except on an unstable fixed point (that is, an indifferent fixed point) at which trajectories are considerably slowed down. This leads to an interplay of chaotic and regular dynamics, a characteristic of intermittent systems [36,39]. From an ergodic theory viewpoint, this phenomenon leads to an absolutely continuous invariant measure having infinite mass. Therefore, standard methods of ergodic theory cannot be applied in this setting; indeed it is well known that Birkhoff's ergodic theorem does not hold under these circumstances, see for instance $[1,2]$.

The first two authors were supported by the German Research Foundation (DFG) grant Renewal Theory and Statistics of Rare Events in Infinite Ergodic Theory (Geschäftszeichen KE 1440/2-1). 
We consider a family $\left\{T_{r}:[0,1] \circlearrowleft\right\}_{r \in[0,1]}$ of Markov interval maps interpolating between the tent map $T_{0}$ and the Farey map $T_{1}$. These interpolating maps, we believe, were first defined in $[10,15]$, and have since attracted much attention. For $r \in[0,1]$, the map $T_{r}:[0,1] \circlearrowleft$ is defined by

$$
T_{r}(x):= \begin{cases}\frac{(2-r) \cdot x}{1-r \cdot x} & \text { if } 0 \leq x \leq 1 / 2, \\ \frac{(2-r) \cdot(1-x)}{1-r+r \cdot x} & \text { if } 1 / 2<x \leq 1 .\end{cases}
$$

For $r \in[0,1)$, many properties of these maps are given in $[10,15]$ and due to the piecewise monotonicity of each $T_{r}$, for $r \in[0,1)$, several results about the associated Perron-Frobenius operator $\mathcal{P}_{r}$, can be deduced from, for instance, $[3,25]$. These latter results can not be applied to the Perron-Frobenius operator $\mathcal{P}_{1}$ of the Farey map $T_{1}$, since any absolutely continuous $T_{1}$-invariant measure is infinite, whereas, for $r \in[0,1)$, there exists a unique absolutely continuous $T_{r}$-invariant probability measure $\mu_{r}$. (See Sect. 2 for the definition of $\mathcal{P}_{r}$.) However, recent advancements have been made on the asymptotic behaviour of $\mathcal{P}_{1}$, see $[24,35]$. (For the role of piecewise monotonicity of the maps $T_{r}$, for $r \in[0,1]$ in our results see Remarks 1 and 6 .)

For $r \in[0,1)$, from the results of [25] it can be deduced that the essential spectral radius of $\mathcal{P}_{r}$ restricted to the Banach space of functions of bounded variation is equal to $1 /(2-r)$. Moreover, in [15], for $r \in[0,1]$, a Hilbert space of analytic functions which is left invariant by each $\mathcal{P}_{r}$ is constructed, and the spectrum of each $\mathcal{P}_{r}$ restricted to this Hilbert space is studied. Here we extend and complement results of $[3,20,25,37]$ on the convergence to equilibrium in one-dimensional systems. In particular, it has been shown, for various classes of regular functions (such as functions of bounded variation and Lipschitz continuous, Hölder continuous, piecewise Hölder continuous and $C^{1+\epsilon}$ functions), that if $f$ belongs to one of these classes then, for $r \in[0,1)$, uniformly on $[0,1]$, we have that

$$
\lim _{n \rightarrow \infty} \mathcal{P}_{r}^{n}(f)=\int f \mathrm{~d} \lambda \cdot h_{r} .
$$

Here $\lambda$ denotes the one-dimensional Lebesgue measure and $h_{r}:=\mathrm{d} \mu_{r} / \mathrm{d} \lambda$. Using arguments similar to those given in [40] one can also prove the above convergence for proper Riemann integrable functions. Applying arguments similar to those presented in $[24,35]$, one can also show that, if $f$ belongs to a certain class of regular functions, then uniformly on compact subsets of $(0,1]$

$$
\lim _{n \rightarrow \infty} \ln (n) \cdot \mathcal{P}_{1}^{n}(f)=\int f \mathrm{~d} \lambda \cdot h_{1} .
$$

One of our main contributions to this theory is given in Theorem 3.1 where we show that the convergence given in (1) also holds for improper Riemann integrable functions with a finite number of singularities and that the type of convergence depends on the structure of the $\omega$-limit set of the singularities with respect to $T_{r}$, for $r \in[0,1)$. We would like to note that also in [7] unbounded observables have been considered in the context of intermittent interval maps 
exhibiting a finite absolutely continuous invariant measure. There the authors focus on probabilistic properties of the dynamical evolution process with respect to these unbounded observables. In a slightly different direction, namely concerning extreme value theory, and in the case that the invariant measure is finite Gouëzel [18] and Tyran-Kaminska [41] have considered stable limit theorems for certain unbounded observables.

We also study the case when $r=1$, for which any absolutely continuous invariant measure has infinite mass. Thaler [40] was the first to discern the asymptotics of the Perron-Frobenius operator of a class of interval maps preserving an infinite measure. This class of maps, to which the Farey map does not belong, have become to be known as Thaler maps. In an effort to generalise this work, by combining renewal theoretical arguments and functional analytic techniques, a new approach to estimate the decay of correlation of a dynamical system was achieved by Sarig [38]. Subsequently, Gouëzel [16,17] generalised these methods. Using these ideas and employing the methods of Garsia and Lamperti [14] and Erickson [9], recently Melbourne and Terhesiu [35] proved a landmark result on the asymptotic rate of convergence of the 'return time operator' (see Sect. 4.2.1) and showed that these result can be applied to Thaler maps, AFN maps, and Pomeau-Manneville maps as well as to maps for which the first return map is Gibbs-Markov. Thus, the question which naturally arises is, whether this asymptotic rate can be related to the asymptotic rate of convergence of iterates of the transfer operator itself and hence the PerronFrobenius operator. This was already partially deduced in [19,24,35], namely, for a specific class observables which are bounded. In this article we present a proof of this result for the Farey map (Theorems 4.9, 4.10) and moreover show that this class of observables can be extended (Theorem 3.2). Indeed, we compute the asymptotic behaviour of the iterates of the Perron-Frobenius operator $\mathcal{P}_{r}$ acting on an observable with a finite number of singularities, and show that the type of convergence depends on the structure of the $\omega$-limit set, with respect to $T_{1}$, of the singularities.

Let us take the opportunity to say a few words on the proofs of our main theorems. The proofs of our results for $r \in[0,1)$ rely on arguments from ergodic theory, for instance those which can be found in $[3,25,37]$, together with the principle of bounded distortion. For the case $r=1$ more sophisticated methods are required. Indeed, we use results of [35] which are based on operator renewal techniques which require Banach spaces with certain properties (see Page 16). To obtain refined results on the set of points of non-convergence, that is to show it is of Hausdorff dimension zero, it is important to choose a Banach space which distinguishes functions pointwise.

We remark that from an ergodic theory point of view the Farey map is of great interest since it is expanding everywhere except at the indifferent fixed point where it has (right) derivative one. This makes the Farey map a simple model of physical phenomena such as intermittency [36]. Further, from the viewpoint of number theory, the Farey map encodes the continued fraction algorithm as well as the Riemann zeta function. In particular, it has an induced version topologically conjugate to the Gauss map [34]. Also, several models of 
statistical mechanics have been considered in recent years in connection to the Farey map and continued fractions [12,30-33].

Finally, we would like to acknowledge that this work has arisen out of our attempts to understand and generalise the work of $[35,40]$.

\subsection{Outline}

In the following section we present essential definitions and state various preliminary results. In Sect. 3 we formally state our results. Several further definitions and preliminary results are given in Sect. 4 . We divide this section into three parts. In the first part we present some properties of functions of bounded variation, the second part contains preliminaries for the case when $r \in[0,1)$ and the third part contains preliminaries for the case when $r=1$. In this latter case, namely when $r=1$, we present two key results (Theorems 4.9, 4.10). These results provide mild conditions under which the asymptotic behaviour of iterates of the Farey transfer operator $\widehat{T}_{1}$ (and hence the Perron-Frobenius operator $\mathcal{P}_{1}$ ) can be deduced from the asymptotic behaviour of the first return time operators. Although, Theorem 4.9 appears in [35], recently a counterexample was given in [24] which shows that this result does not hold in the full generality as stated in [35]. Thus, here we present a full proof of this result. Further, in the case that $r=1$, we will make use of [35, Theorem 2.1] for which we require the existence of a Banach space with certain properties. Such a Banach space is described in Proposition 4.8. In Sect. 5 we give the proofs of our main results, Theorems 3.1, 3.2 and 3.3 .

\subsection{Notation}

The natural numbers will be denoted by $\mathbb{N}$, the real numbers by $\mathbb{R}$ and the complex numbers by $\mathbb{C}$. We will also use the symbol $\mathbb{N}_{0}$ to denote the set of non-negative integers, $\mathbb{R}^{+}$to denote the set of positive real numbers and $\overline{\mathbb{R}}$ to denote the extended real numbers, namely $\overline{\mathbb{R}}=\mathbb{R} \cup\{ \pm \infty\}$.

Following convention, we use the symbol $\sim$ between the elements of two sequences of real or complex numbers $\left(b_{n}\right)_{n \in \mathbb{N}}$ and $\left(c_{n}\right)_{n \in \mathbb{N}}$ to mean that the sequences are asymptotically equivalent, namely that $\lim _{n \rightarrow+\infty} b_{n} / c_{n}=1$, and we use the Landau notation $b_{n}=\mathfrak{o}\left(c_{n}\right)$ if $\lim _{n \rightarrow+\infty} b_{n} / c_{n}=0$. The same notation is used between two $\mathbb{R}$-valued or $\mathbb{C}$-valued functions $f$ and $g$; that is, if $\lim _{x \rightarrow+\infty} f(x) / g(x)=0$, then we write $f=\mathfrak{o}(g)$.

\section{Central Definitions}

For $r \in(0,1]$, the map $T_{r}$ has two fixed points, one at zero and one at $1-(3-$ $\sqrt{9-4 r}) /(2 \cdot r)$. For $r=0$, the map $T_{0}$ has one fixed point at zero and the other at $2 / 3$. The inverse branches $f_{r, 0}, f_{r, 1}:[0,1] \circlearrowleft$ of $T_{r}$ are given by

$$
f_{r, 0}(x):=\frac{x}{2-r+r \cdot x} \quad \text { and } \quad f_{r, 1}(x):=\frac{1+(1-r) \cdot(1-x)}{2-r+r \cdot x} .
$$


In $[15,28]$ it was shown that the absolutely continuous invariant measure $\mu_{r}$ of $T_{r}$ is given by

$$
h_{r}(x):=\frac{\mathrm{d} \mu_{r}}{\mathrm{~d} \lambda}(x)= \begin{cases}1 & \text { if } r=0, \\ \frac{-r}{\ln (1-r)} \frac{1}{1-r+r \cdot x} & \text { if } r \in(0,1), \\ 1 / x & \text { if } r=1 .\end{cases}
$$

We let $\mathcal{L}_{r}^{1}([0,1])$ denote the Banach space of equivalence classes $[f]$ of functions, where for each representative $f:[0,1] \rightarrow \mathbb{C}$ of $[f]_{r}$,

$$
\|f\|_{r, 1}:=\int|f| \mathrm{d} \mu_{r}<+\infty
$$

and where $f, g$ belong to the same equivalence class, if and only if,

$$
\|f-g\|_{r, 1}=0 \text {. }
$$

Throughout, following convention, we write $f \in \mathcal{L}_{r}^{1}([0,1])$ to mean a function $f:[0,1] \rightarrow \mathbb{C}$ which belongs to an equivalence class of $\mathcal{L}_{r}^{1}([0,1])$.

For $r \in[0,1]$, the Perron-Frobenius operator $\mathcal{P}_{r}: \mathcal{L}_{0}^{1}([0,1]) \circlearrowleft$ of $T_{r}$ is defined, for $f \in \mathcal{L}_{0}^{1}([0,1])$, by

$$
\mathcal{P}_{r}(f)=\left|f_{r, 0}^{\prime}\right| \cdot f \circ f_{r, 0}+\left|f_{r, 1}^{\prime}\right| \cdot f \circ f_{r, 1} .
$$

Here $f_{r, 0}^{\prime}$ and $f_{r, 1}^{\prime}$ denote the derivative of the contractions $f_{r, 0}$ and $f_{r, 1}$, respectively. Note, the domain of definition of $\mathcal{P}_{r}$ can be extended to any welldefined $\mathbb{C}$-valued or $\overline{\mathbb{R}}$-valued function. In $[15,28]$ it has been shown that $h_{r}$ is the unique fixed point function of $\mathcal{P}_{r}$, namely that $\mathcal{P}_{r}\left(h_{r}\right)=h_{r}$, and so

$$
\mathcal{P}_{r}(f):=\frac{\mathrm{d} \nu_{f} \circ T_{r}^{-1}}{\mathrm{~d} \lambda}, \quad \text { where } \nu_{f}(A):=\int \mathbb{1}_{A} \cdot f \mathrm{~d} \lambda,
$$

for all Borel sets $A \subset[0,1]$.

Two important function spaces which we will use are defined below.

1. The space $\operatorname{BV}(0,1)$ which is defined to be the set of right-continuous functions $f:[0,1] \rightarrow \mathbb{C}$ such that the norm $\|f\|_{\mathrm{BV}}:=V_{[0,1]}(f)+\|f\|_{\infty}$ is finite. Here $V_{[0,1]}(f)$ denotes the variation of $f$ and $\|f\|_{\infty}$ denotes the supremum of $|f|$ and is defined by $\|f\|_{\infty}:=\sup \{|f(x)|: x \in[0,1]\}$. Letting $a, b \in \mathbb{R}$, we recall that the variation of a function $f:[a, b] \rightarrow \mathbb{C}$ is defined by

$$
V_{[a, b]}(f):=\sup _{P}\left\{\sum_{k=1}^{n}\left|f\left(x_{k}\right)-f\left(x_{k-1}\right)\right|\right\} .
$$

Here the supremum is taken over finite partitions $P:=\left\{I_{i}=\left[x_{i-1}, x_{i}\right]\right.$ : $i \in\{1,2, \ldots, n\}\}$, where $a:=x_{0}<x_{1}<\cdots<x_{n-1}<x_{n}:=b$, is a chain of points belonging to $[a, b]$, for some $n \in \mathbb{N}$. We say that a function $f:[a, b] \rightarrow \mathbb{R}$ is of bounded variation if $V_{[a, b]}(f)$ is finite.

2. The space $\mathfrak{U}_{\beta, \alpha}$ is defined for $\alpha \in(0,1)$ and $\beta \in[0,1]$, and where $v:[0,1] \rightarrow \overline{\mathbb{R}}$ belongs to $\mathfrak{U}_{\beta, \alpha}$ if and only if

(a) $\lim _{x \uparrow \beta} v(x)=\lim _{x \downarrow \beta} v(x)=+\infty$, 
(b) for any compact set $K \subset[0,1] \backslash\{\beta\}$, we have $v \cdot \mathbb{1}_{K} \in \operatorname{BV}(0,1)$, where for a set $A \subset[0,1]$ we let $\mathbb{1}_{A}:[0,1] \rightarrow \mathbb{R}$ denote the characteristic function on $A$, namely $\mathbb{1}_{A}(x)=1$, for $x \in A$, and 0 , otherwise.

(c) there exists a connected open neighbourhood $U \subset[0,1]$ of $\beta$, under the (Euclidean) subspace topology, and two constants $C_{1}, C_{2}$ such that $C_{1}|\beta-x|^{-\alpha} \leq v(x) \leq C_{2}|\beta-x|^{-\alpha}$, for all $x \in U$.

Note conditions (b) and (c) immediately imply that if $v \in \mathfrak{U}_{\beta, \alpha}$, then $v$ is improper Riemann integrable. Moreover, without loss of generality, throughout we assume that $v$ is positive.

Define the $\omega$-limit set of $\beta \in[0,1]$ with respect to $T_{r}$ to be the set of accumulation points of the orbit $\left(T_{r}^{n}(\beta)\right)_{n \in \mathbb{N}_{0}}$ and denote it by

$$
\omega_{r}(\beta):=\bigcap_{k \in \mathbb{N}_{0}} \overline{\left\{T_{r}^{\ell}(\beta): \ell \geq k\right\}} .
$$

We say that a point $x \in[0,1]$ is pre-periodic with respect to $T_{r}$ if there exist $m \in \mathbb{N}$ and $n \in \mathbb{N}_{0}$ with

$$
T_{r}^{n+k}(x)=T_{r}^{n+m+k}(x),
$$

for all $k \in \mathbb{N}_{0}$. Indeed, for $r \in(0,1]$, we have that $1-(3-\sqrt{9-4 \cdot r}) /(2 \cdot r)$ is pre-periodic with respect to $T_{r}$. For a given pre-periodic point $x$ with respect to $T_{r}$, we define the period length of $x$ to be the minimal $m$ such that the equality in (2) holds.

In the case when $r=1$, as mentioned above, the map $T_{1}$ is the celebrated Farey map which encodes the continued fraction expansion algorithm. A continued fraction expansion of an irrational $\beta \in[0,1]$ is denoted by $\left[0 ; a_{1}, a_{2}, \ldots\right]$ where

$$
\beta=\frac{1}{a_{1}+\frac{1}{a_{2}+\cdots}}
$$

and $a_{n} \in \mathbb{N}$, for all $n \in \mathbb{N}$. The continued fraction expansion of a rational $\beta \in[0,1]$ is denoted by $\left[0 ; a_{1}, a_{2}, \ldots, a_{k}\right]$, where

$$
\beta=\frac{1}{a_{1}+\frac{1}{\cdots+\frac{1}{a_{k}}}},
$$

$a_{n} \in \mathbb{N}$, for all $n \in\{1,2, \ldots, k-1\}$ and $a_{k} \in \mathbb{N} \backslash\{1\}$, except in the case that $\beta=1$ when $k=1$ and $a_{k}=1$. If there exist $m \in \mathbb{N}_{0}$ and $n \in \mathbb{N}$ such that $a_{m+k}=a_{m+k+n+1}$, for all $k \in \mathbb{N}$, then we write

$\beta=\left[0 ; a_{1}, a_{2}, \ldots, a_{m}, \overline{a_{m+1}}, a_{m+2}, \ldots, a_{m+n}\right]$.

For $\beta \in[0,1]$, we let $p_{n}=p_{n}(\beta)$ and $q_{n}=q_{n}(\beta)$ be defined recursively by

$$
p_{n}:=a_{n} p_{n-1}+p_{n-2}, \quad \text { and } \quad q_{n}:=a_{n} q_{n-1}+q_{n-2},
$$


where $p_{-1}:=1, q_{-1}:=0, p_{0}:=0, q_{0}:=1$. Note, for $n \in \mathbb{N}$, that

$$
\frac{p_{n}}{q_{n}}=\left[0 ; a_{1}, a_{2}, \ldots, a_{n}\right] \text { and } p_{n-1} \cdot q_{n}-p_{n} \cdot q_{n-1}=1,
$$

and if $\beta=\left[0 ; a_{1}, a_{2}, \ldots, a_{n}\right]$ is rational, then we set $a_{m}=0$ for all $m>n$. Given $\alpha \in(0,1)$ we say that an irrational $\beta=\left[0 ; a_{1}, a_{2}, \ldots\right] \in[0,1]$ is of intermediate $\alpha$-type if and only if there exists an $\epsilon>0$, with

$$
\sum_{n=1}^{+\infty} \sum_{j=1}^{a_{n}}\left(t_{n, j}\right)^{-2 \cdot(1-\alpha)+\epsilon}<+\infty
$$

where $s_{n, j} / t_{n, j}=\left[0 ; a_{1}, \ldots, a_{n-1}, j\right]$ and where $s_{n, j}, t_{n, j} \in \mathbb{N}$ are co-prime. (Using the terminology from continued fraction expansion one refers to $s_{n, j} / t_{n, j}$ as an intermediate approximant to $\beta$.) We also note the following:

1. If $\beta$ is pre-periodic, or more generally, if the continued fraction entries $a_{i}$ of $\beta$ are bounded, then $\beta$ is of intermediate $\alpha$-type, for all $\alpha \in(0,1)$.

2. If $\alpha<1 / 2$, then every irrational $\beta$, is of intermediate $\alpha$-type.

3. It follows from the results of [29] that

$\operatorname{dim}_{\mathcal{H}}(\{\beta \in[0,1]: \beta$ is of intermediate $\alpha$-type for all $\alpha \in(0,1)\})=1$.

Here and throughout we will denote the Hausdorff dimension of a set $A \subset \mathbb{R}$ by $\operatorname{dim}_{\mathcal{H}}(A)$, see [11] for the definition and further details on the Hausdorff dimension of a set.

\section{Main Results}

\subsection{The Case $r \in[0,1)$. Finite Absolutely Continuous Invariant Measure}

Theorem 3.1. For $r \in[0,1)$, if $\alpha \in(0,1)$ and $\beta \in[0,1]$, then, for each $v \in \mathfrak{U}_{\beta, \alpha}$, we have that

$$
\lim _{n \rightarrow \infty} \mathcal{P}_{r}^{n}(v)=\int v \mathrm{~d} \lambda \cdot h_{r},
$$

uniformly on compact subsets of $[0,1] \backslash \omega_{r}(\beta)$ and pointwise outside a set with Hausdorff dimension equal to zero. If $\beta \in[0,1]$ is pre-periodic with respect to $T_{r}$ and has period length strictly greater than one, then on the finite set $\omega_{r}(\beta)$ we have that

$$
\liminf _{n \rightarrow+\infty} \mathcal{P}_{r}^{n}(v)=\int v \mathrm{~d} \lambda \cdot h_{r} \quad \text { and } \quad \limsup _{n \rightarrow+\infty} \mathcal{P}_{r}^{n}(v)=+\infty .
$$

In the case that $\beta \in[0,1]$ is pre-periodic with respect to $T_{r}$ and has period length equal to one then on the singleton $\omega_{r}(\beta)$ we have that the limit in (4) is equal to $+\infty$.

Remark 1. Theorem 3.1 holds for more general systems, for instance expanding piecewise $C^{1+\epsilon}$ Markov interval maps $T$ : $[0,1] \circlearrowleft$. The proof of such a result follows in the same manner as those set out below. 


\subsection{The Case $r=1$. Infinite Absolutely Continuous Invariant Measure}

Theorem 3.2. If $\alpha \in(0,1)$ and if $\beta \in(0,1]$ is either rational or irrational of intermediate $\alpha$-type, then, for each $v \in \mathfrak{U}_{\beta, \alpha}$, we have that

$$
\lim _{n \rightarrow \infty} \ln (n) \cdot \mathcal{P}_{1}^{n}(v)=\int_{[0,1]} v \mathrm{~d} \lambda \cdot h_{1},
$$

uniformly on compact subsets of $(0,1) \backslash \omega_{1}(\beta)$ and pointwise outside a set with Hausdorff dimension equal to zero. If $\beta \in(0,1]$ is pre-periodic with respect to $T_{1}$ and has period length strictly greater than one, then on the finite set $\omega_{1}(\beta)$ we have that

$$
\liminf _{n \rightarrow+\infty} \ln (n) \cdot \mathcal{P}_{1}^{n}(v)=\int v \mathrm{~d} \lambda \cdot h_{1} \quad \text { and } \quad \limsup _{n \rightarrow+\infty} \ln (n) \cdot \mathcal{P}_{1}^{n}(v)=+\infty .
$$

In the case that $\beta \in(0,1]$ is a noble number, that is a pre-periodic number with respect to $T_{1}$ and has period length equal to one, then on the singleton $\omega_{1}(\beta)$ we have that the limit in (6) is equal to $+\infty$.

Remark 2. It is well known that the term $\ln (n)$ in (6) and (7) defines a sequence asymptotic to the wandering rate of the Farey map $T_{1}$. Indeed this term is well defined for any interval map $T:[0,1] \circlearrowleft$ and for the maps we are concerned with it is given by

$$
w_{n}\left(T_{r}\right):=\mu_{r}\left(\bigcup_{k=0}^{n-1} T_{r}^{-k}([1 / 2,1])\right) .
$$

Indeed from this definition one sees that for $r \in[0,1)$ we have that $w_{n}\left(T_{r}\right) \sim 1$ and for $r=1$ we have that $w_{n}\left(T_{r}\right) \sim \ln (n)$.

Remark 3. We highlight an interesting difference between Theorems 3.1 and 3.2, which is a result of the Farey map having an indifference fixed point at zero. In the case that $r \in[0,1), \alpha \in(0,1), \beta$ is an $r$-rational (see Sect. 4) and $v \in \mathfrak{U}_{\beta, \alpha}$, we have that

$$
\lim _{n \rightarrow \infty} \mathcal{P}_{r}^{n}(v)(0)=+\infty
$$

whereas, for $r=1, \alpha \in(0,1), \beta$ is a rational number and $v \in \mathfrak{U}_{\beta, \alpha}$, we have that

$$
\lim _{n \rightarrow \infty} \ln (n) \cdot \mathcal{P}_{1}^{n}(v)(0)=0 .
$$

(Note that the points $0,1 / 2$ and 1 are $r$-rationals for all $r \in[0,1]$.)

Remark 4 . In the case that one replaces the norm $\|\cdot\|_{\infty}$ by the essential supremum norm in the definition of $\operatorname{BV}(0,1)$, and hence $\mathfrak{U}_{\beta, \alpha}$, the limit in (6) holds uniformly Lebesgue almost everywhere on compact subsets of $(0,1) \backslash \omega_{1}(\beta)$ and pointwise Lebesgue almost everywhere on $(0,1)$.

Remark 5. Concerning the results given in (5) of Theorems 3.1 and (7) of Theorem 3.2, we note that they do not immediately follow from [35, Theorem 
10.5]. Indeed, in the proofs of (5) and (7) we show that along sub-sequences $\left(k_{n}\right)_{n \in \mathbb{N}}$ and $\left(j_{n}\right)_{n \in \mathbb{N}}$ of $\mathbb{N}$ both with positive density, we have that

$$
\lim _{n \rightarrow+\infty} \mathcal{P}_{r}^{j_{n}}(v)=\int v \mathrm{~d} \lambda \cdot h_{r} \text { and } \lim _{n \rightarrow+\infty} \mathcal{P}_{r}^{k_{n}}(v)=+\infty,
$$

for $r \in[0,1)$, and

$$
\lim _{n \rightarrow+\infty} \ln \left(j_{n}\right) \cdot \mathcal{P}_{1}^{j_{n}}(v)=\int v \mathrm{~d} \lambda \cdot h_{1} \quad \text { and } \quad \lim _{n \rightarrow+\infty} \ln \left(k_{n}\right) \cdot \mathcal{P}_{1}^{k_{n}}(v)=+\infty
$$

pointwise on the associated $\omega$-limit set. On the other hand, the results of [35, Theorem 10.5] show a convergence result for a class of bounded observables along subsequences outside a zero density set.

In the following theorem, for the observable $v_{\beta, \alpha}(x)=|\beta-x|^{-\alpha}$, we demonstrate that on the $\omega$-limit set the values of the limit superior depend on the Diophantine properties of $\beta$.

Theorem 3.3. (a) There exist non-periodic $\beta$ and $\varrho \in(0,1]$ both with bounded continued fraction entries but such that, on the one hand, for all $\alpha \in$ $(0,1)$, on $\omega_{1}(\beta)$, we have

$$
\lim _{n \rightarrow+\infty} \ln (n) \cdot \mathcal{P}_{1}^{n}\left(v_{\beta, \alpha}\right)=\int v_{\beta, \alpha} \mathrm{d} \lambda \cdot h_{1},
$$

and on the other hand, for all $\alpha \in(0,1 / 2)$, on $\omega_{1}(\varrho)$, we have

$$
\lim _{n \rightarrow \infty} \ln (n) \cdot \mathcal{P}_{1}^{n}\left(v_{\varrho, \alpha}\right)=\int v_{\varrho, \alpha} \mathrm{d} \lambda \cdot h_{1} ;
$$

otherwise, for all $\alpha \in(1 / 2,1)$, on $\omega_{1}(\varrho)$, we have

$$
\begin{aligned}
& \liminf _{n \rightarrow+\infty} \ln (n) \cdot \mathcal{P}_{1}^{n}\left(v_{\varrho, \alpha}\right)=\int v_{\varrho, \alpha} \mathrm{d} \lambda \cdot h_{1} \\
& \text { and } \\
& \limsup _{n \rightarrow+\infty} \ln (n) \cdot \mathcal{P}_{1}^{n}\left(v_{\varrho, \alpha}\right)=+\infty .
\end{aligned}
$$

(b) Let $\alpha \in(0,1)$ and let $\beta=\left[0 ; a_{1}, a_{2}, \ldots\right] \in(0,1]$ be of intermediate $\alpha$-type such that

$$
\lim _{n \rightarrow+\infty} a_{n}=+\infty .
$$

Fix $k \in \mathbb{N}$ and let $l=l(k):=\min \left\{i \in \mathbb{N}: a_{m} \geq k\right.$ for all $\left.m \geq i\right\}$. For all $j \geq l$, set $n_{k, j} \in \mathbb{N}$ to be the unique integer satisfying

$$
T_{1}^{n_{k, j}}(\beta)=\left[0 ; k, a_{j+1}, a_{j+2}, \ldots\right]
$$

and set

$$
\mathscr{S}_{k, j}:=\frac{\left(a_{j+1}\right)^{\alpha} \cdot \ln \left(n_{k, j}\right)}{\left(q_{j}\right)^{2 \cdot(1-\alpha)}},
$$

where $q_{n}$ is as defined in (3). If $\limsup _{j \rightarrow \infty} \mathscr{S}_{k, j}=0$, then

$$
\lim _{n \rightarrow+\infty} \ln (n) \cdot \mathcal{P}_{1}^{n}\left(v_{\beta, \alpha}\right)(1 / k)=\int v_{\beta, \alpha} \mathrm{d} \lambda \cdot h_{1}
$$


otherwise,

$$
\begin{gathered}
\liminf _{n \rightarrow+\infty} \ln (n) \cdot \mathcal{P}_{1}^{n}\left(v_{\beta, \alpha}\right)(1 / k)=\int v_{\beta, \alpha} \mathrm{d} \lambda \cdot h_{1} \text { and } \\
\limsup _{n \rightarrow+\infty} \ln (n) \cdot \mathcal{P}_{1}^{n}\left(v_{\beta, \alpha}\right)(1 / k)>\int v_{\beta, \alpha} \mathrm{d} \lambda \cdot h_{1} .
\end{gathered}
$$

(Note that in this case $\omega_{1}(\beta)=\{1 / n: n \in \mathbb{N}\} \cup\{0\}$.)

Remark 6. Analogous results concerning Theorems 3.2 and 3.3, we believe will hold true for more general systems with an infinite absolutely continuous invariant measure, for instance the $\delta$-expansive $\alpha$-Farey maps considered in [24]. (Here, one would replace the conditions on the continued fraction expansion, with conditions on the $\alpha$-Lüroth expansion, see [27].) Indeed, in the case of the Farey map $T_{1}$, calculations simplify through exploiting the natural underlying coding in terms of continued fractions.

\section{Preliminaries}

We let $\Sigma:=\{0,1\}, \Sigma^{n}:=\{0,1\}^{n}$, for $n \in \mathbb{N}$, and let $\Sigma^{\mathbb{N}}$ denote the set of all infinite words over the alphabet $\Sigma$. For $\beta \in[0,1]$ we let $\vartheta_{r}(\beta)$ denote the infinite word $\left(\vartheta_{r, 1}(\beta), \vartheta_{r, 2}(\beta), \ldots\right) \in \Sigma^{\mathbb{N}}$, where

$$
\vartheta_{r, n}(\beta):= \begin{cases}0 & \text { if } T_{r}^{n-1}(\beta) \leq 1 / 2, \\ 1 & \text { otherwise. }\end{cases}
$$

Unless otherwise stated, let $n \in \mathbb{N}$ be fixed. For $\vartheta=\left(\vartheta_{1}, \vartheta_{2}, \ldots\right) \in \Sigma^{\mathbb{N}}$, we set $\left.\vartheta\right|_{n}:=\left(\vartheta_{1}, \ldots, \vartheta_{n}\right) \in \Sigma^{n}$ and, for $\varphi=\left(\varphi_{1}, \varphi_{2}, \ldots, \varphi_{n}\right) \in \Sigma^{n}$, we set $f_{r, \varphi}:=f_{r, \varphi_{1}} \circ \cdots \circ f_{r, \varphi_{n}}$ and $[\varphi]_{r}:=f_{r, \varphi}([0,1])$. The set $[\varphi]_{r}$ is referred to as a cylinder set of length $n$ with respect to $T_{r}$. We let $\left.\vartheta_{r}^{ \pm}(\beta)\right|_{n} \in \Sigma^{n}$ denote unique finite words such that

$$
\left[\left.\vartheta_{r}^{+}(\beta)\right|_{n}\right]_{r} \cap\left[\left.\vartheta_{r}(\beta)\right|_{n}\right]_{r} \neq \emptyset, \quad\left[\left.\vartheta_{r}^{-}(\beta)\right|_{n}\right]_{r} \cap\left[\left.\vartheta_{r}(\beta)\right|_{n}\right]_{r} \neq \emptyset
$$

and such that either one of the following sets of inequalities hold,

$$
f_{\left.\vartheta_{r}^{-}(\beta)\right|_{n}}(x) \leq f_{\left.\vartheta_{r}(\beta)\right|_{n}}(x)<f_{\left.\vartheta_{r}^{+}(\beta)\right|_{n}}(x)
$$

or

$$
f_{\left.\vartheta_{r}^{-}(\beta)\right|_{n}}(x)<f_{\left.\vartheta_{r}(\beta)\right|_{n}}(x) \leq f_{\left.\vartheta_{r}^{+}(\beta)\right|_{n}}(x),
$$

for $x \in(0,1)$. Note that in the case when there exists $\vartheta \in \Sigma^{m}$, for some $m \in \mathbb{N}$, such that either $f_{r, \vartheta}(0)=\beta$ or $f_{r, \vartheta}(1)=\beta$, then it can occur that $\left.\vartheta_{r}^{+}(\beta)\right|_{m}=$ $\left.\vartheta_{r}(\beta)\right|_{m}$ or that $\left.\vartheta_{r}^{-}(\beta)\right|_{m}=\left.\vartheta_{r}(\beta)\right|_{m}$. We call such points $r$-rationals. (Note, if $r=1$, then the set of $r$-rationals is precisely the set of rational numbers in the closed unit interval $[0,1]$.) For ease of notation, we set

$$
\mathfrak{W}_{r, n}(\beta):=\left\{\left.\vartheta_{r}^{-}(\beta)\right|_{n},\left.\vartheta_{r}(\beta)\right|_{n},\left.\vartheta_{r}^{+}(\beta)\right|_{n}\right\}
$$

and

$$
\left[W_{r, n}(\beta)\right]=\left[\left.\vartheta_{r}^{-}(\beta)\right|_{n}\right]_{r} \cup\left[\left.\vartheta_{r}(\beta)\right|_{n}\right]_{r} \cup\left[\left.\vartheta_{r}^{+}(\beta)\right|_{n}\right]_{r}
$$


Lemma 4.1. Let $r \in[0,1]$ and $n \in \mathbb{N}$ be fixed. If $\vartheta=\left(\vartheta_{1}, \vartheta_{2}, \ldots, \vartheta_{n}\right)$ and $\nu=\left(\nu_{1}, \nu_{2}, \ldots, \nu_{n}\right)$ denote two distinct elements of $\Sigma^{n}$, with $[\vartheta]_{r} \cap[\nu]_{r} \neq \emptyset$, then there exists a unique $i \in\{1,2, \ldots, n\}$ such that $\vartheta_{i} \neq \nu_{i}$ and $\vartheta_{j}=\nu_{j}$ for all $j \in\{1,2, \ldots, n\} \backslash\{i\}$.

Proof. For $n=1$ we have that $[(0)]_{r}=f_{r, 0}([0,1])=[0,1 / 2]$ and $[(1)]_{r}=$ $f_{r, 1}([0,1])=[1 / 2,1]$. We now proceed by induction on $n$. Suppose the statement is true for some $n \in \mathbb{N}$. Let $\vartheta=\left(\vartheta_{1}, \vartheta_{2}, \ldots, \vartheta_{n+1}\right)$ and $\nu=\left(\nu_{1}, \nu_{2}, \ldots\right.$, $\left.\nu_{n+1}\right)$ denote two distinct elements of $\Sigma^{n+1}$, with $[\vartheta]_{r} \cap[\nu]_{r} \neq \emptyset$. We have two cases to consider, namely, if there exists an $\xi \in \Sigma^{n}$ such that $[\vartheta]_{r} \cup[\nu]_{r}=[\xi]_{r}$, or not.

In the case that there exists $\xi=\left(\xi_{1}, \ldots, \xi_{n}\right) \in \Sigma^{n}$ with $[\vartheta]_{r} \cup[\nu]_{r}=[\xi]_{r}$, then, by construction, either

1. $\vartheta=\left(\xi_{1}, \xi_{2}, \ldots, \xi_{n}, 0\right)$ and $\nu=\left(\xi_{1}, \xi_{2}, \ldots, \xi_{n}, 1\right)$, or

2. $\vartheta=\left(\xi_{1}, \xi_{2}, \ldots, \xi_{n}, 1\right)$ and $\nu=\left(\xi_{1}, \xi_{2}, \ldots, \xi_{n}, 0\right)$,

in which case the result follows.

In the case that there does not exist an $\xi \in \Sigma^{n}$ with $[\vartheta]_{r} \cup[\nu]_{r}=[\xi]_{r}$, then, by construction, there exist $\xi=\left(\xi_{1}, \xi_{2}, \ldots, \xi_{n}\right), \eta=\left(\eta_{1}, \eta_{2}, \ldots, \eta_{n}\right) \in$ $\Sigma^{n}$ such that $[\xi]_{r} \cap[\eta]_{r} \neq \emptyset,[\vartheta]_{r} \subset[\xi]_{r}$ and $[\nu]_{r} \subset[\eta]_{r}$. Therefore, by the inductive hypothesis, we have that either $f_{r, \xi}$ is order preserving and $f_{r, \eta}$ is order reversing, or $f_{r, \xi}$ is order reversing and $f_{r, \eta}$ is order preserving. Assuming the former of these two cases, by construction we have that $\vartheta=\left(\xi_{1}, \ldots, \xi_{n}, 1\right)$ and $\nu=\left(\eta_{1}, \ldots, \eta_{n}, 1\right)$, in which case the result follows. In the remaining case, namely that $f_{r, \xi}$ is order reversing and $f_{r, \eta}$ is order preserving, by construction we have that $\vartheta=\left(\xi_{1}, \ldots, \xi_{n}, 0\right)$ and $\nu=\left(\eta_{1}, \ldots, \eta_{n}, 0\right)$, which concludes the proof.

Definition 4.1. Given $r \in[0,1], \alpha \in(0,1)$ and $\beta \in[0,1]$, we define the $r$-tail of the observable $v_{\beta, \alpha}: x \mapsto|x-\beta|^{-\alpha}$ by

$$
\begin{aligned}
v^{n, r} & =v_{\beta, \alpha, n, r}:=\mathcal{P}_{r}^{n}\left(v_{\beta, \alpha} \cdot \mathbb{1}_{\left[W_{r, n}(\beta)\right]}\right) \\
& = \begin{cases}\sum_{\vartheta \in \mathfrak{W}_{r, n}(\beta)}\left|f_{r, \vartheta}^{\prime}(x)\right| \cdot v_{\beta, \alpha} \circ f_{r, \vartheta} & \text { if } r \in[0,1), \\
\left|f_{r,\left.\vartheta_{1}(\beta)\right|_{n}}^{\prime}(x)\right| \cdot v_{\beta, \alpha} \circ f_{r,\left.\vartheta_{1}(\beta)\right|_{n}} & \text { if } r=1 .\end{cases}
\end{aligned}
$$

Further, for $r \in[0,1], \alpha \in(0,1), \beta \in[0,1], n \in \mathbb{N}$ and $\eta>0$ set

$$
A_{n, r, \eta}:= \begin{cases}\left\{x \in[0,1]: v^{n, r}(x)>\eta\right\} & \text { if } r \in[0,1), \\ \left\{x \in[0,1]: \ln (n) \cdot v^{n, r}(x)>\eta\right\} & \text { if } r=1 .\end{cases}
$$

\subsection{Auxiliary Results for the Case $r \in[0,1)$}

\subsubsection{Bounded Distortion.}

Lemma 4.2. ([26, Lemma 3.2] Bounded Distortion) Let $r \in[0,1)$ be fixed. There exists a sequence $\left(\varrho_{n}\right)_{n \in \mathbb{N}_{0}}$, dependent on $r$, with $\varrho_{n}>0$ for each $n \in \mathbb{N}_{0}$ and $\lim _{n \rightarrow+\infty} \varrho_{n}=1$, such that, for all $m, n \in \mathbb{N}_{0}, \vartheta \in \Sigma^{m}, \varphi \in \Sigma^{n}$ and $x, y \in[\vartheta]_{r}$, 
we have that

$$
\varrho_{m}^{-1} \leq\left|\frac{f_{r, \varphi}^{\prime}(x)}{f_{r, \varphi}^{\prime}(y)}\right| \leq \varrho_{m}
$$

(Here $\Sigma^{0}$ denotes the set containing the empty set and $f_{r, \emptyset}$ denotes the identity function $[0,1] \ni x \mapsto x$.)

Lemma 4.3. Let $n \in \mathbb{N}$ be fixed. If $\vartheta=\left(\vartheta_{1}, \vartheta_{2}, \ldots, \vartheta_{n}\right)$ and $\nu=\left(\nu_{1}, \nu_{2}, \ldots, \nu_{n}\right)$ denote two distinct elements of $\Sigma^{n}$, with $[\vartheta] \cap[\nu] \neq \emptyset$, then there exists $a$ positive constant $K$ such that, for all $x, y \in[0,1]$,

$$
K^{-1} \leq\left|\frac{f_{r, \vartheta}^{\prime}(x)}{f_{r, \nu}^{\prime}(y)}\right| \leq K
$$

Proof. This is a consequence of the chain rule and Lemmata 4.1 and 4.2.

\subsubsection{Classical Results on Convergence to Equilibrium.}

Theorem 4.4. $[3,5,25,37]$ For $r \in[0,1)$ there exist constants $M=M(r)>0$ and $p=p(r) \in(0,1)$ such that, for all $f \in \mathrm{BV}(0,1)$,

$$
\left\|\mathcal{P}_{r}^{n}(f)-\int f \mathrm{~d} \lambda \cdot h_{r}\right\|_{\mathrm{BV}} \leq M \cdot p^{n} \cdot\|f\|_{\mathrm{BV}}
$$

Lemma 4.5. For $r \in[0,1), \alpha \in(0,1), \beta \in[0,1]$ and $v \in \mathfrak{U}_{\beta, \alpha}$ we have, uniformly on $[0,1]$, that

$$
\lim _{n \rightarrow \infty} \mathcal{P}_{r}^{n}\left(v \cdot \mathbb{1}_{[0,1] \backslash\left[W_{r, n}(\beta)\right]}\right)=\int v \mathrm{~d} \lambda \cdot h_{r}
$$

Proof. Let $N \in \mathbb{N}$ be fixed. Since $v \cdot \mathbb{1}_{\left.[0,1] \backslash\left[\left.W_{r}(\beta)\right|_{N}\right]\right)} \in \operatorname{BV}(0,1)$, by Theorem 4.4, we have that

$$
\lim _{n \rightarrow+\infty} \mathcal{P}_{r}^{n}\left(v \cdot \mathbb{1}_{\left.[0,1] \backslash\left[\left.W_{r}(\beta)\right|_{N}\right]\right)}\right)=\int v \cdot \mathbb{1}_{\left.[0,1] \backslash\left[\left.W_{r}(\beta)\right|_{N}\right]\right)} \mathrm{d} \lambda \cdot h_{r}
$$

uniformly on $[0,1]$. We will shortly show, with the aid of Lemmeta 4.1 and 4.2 , that, uniformly on $[0,1]$, there exists a positive constant $K \in \mathbb{R}$ such that for all $x \in[0,1]$

$$
\lim _{n \rightarrow+\infty} \mathcal{P}_{r}^{n}\left(v \cdot \mathbb{1}_{\left.\left[\left.W_{r}(\beta)\right|_{N}\right] \backslash\left[\left.W_{r}(\beta)\right|_{n}\right]\right)}\right)(x) \leq K \sum_{k=N}^{+\infty}(2-r)^{-k(1-\alpha)} .
$$

As $v$ is improper Riemann integrable and as $\lim _{N \rightarrow+\infty} \lambda\left(\left[\left.\vartheta_{r}(\beta)\right|_{N}\right]\right)=0$, we have that

$$
\lim _{N \rightarrow+\infty} \int v \cdot \mathbb{1}_{\left.[0,1] \backslash\left[\left.W_{r}(\beta)\right|_{N}\right]\right)} \mathrm{d} \lambda=\int v \mathrm{~d} \lambda
$$

and by the properties of geometric series we have that

$$
\lim _{N \rightarrow+\infty} \sum_{k=N}^{+\infty}(2-r)^{-k}=0 .
$$

Thus assuming the inequality given in (10), since $\mathcal{P}_{r}$ is a positive linear operator and since $N$ was chosen arbitrarily, the result follows. 
We now show the inequality stated in (10). Let $U \subset[0,1]$ be an open set and let $C_{2}$ be a constant such that Condition (c) in the definition of $\mathfrak{U}_{\beta, \alpha}$ is satisfied. Let $n>N \geq 2$ with $\left[\left.W_{r}(\beta)\right|_{N}\right] \subseteq U$ be fixed. For all $x \in[0,1]$, we have that

$$
(2-r) / 4 \leq\left|f_{r, 0}^{\prime}(x)\right|,\left|f_{r, 1}^{\prime}(x)\right| \leq 1 /(2-r) .
$$

This in tandem with Lemmata 4.2 and 4.3 and the mean value theorem, gives that there exists a positive constant $\varrho \in \mathbb{R}$ such that the following chain of inequalities hold, for all $x \in[0,1]$.

$$
\begin{aligned}
\mathcal{P}_{r}^{n} & \left(v \cdot \mathbb{1}_{\left.\left[\left.W_{r}(\beta)\right|_{N}\right] \backslash\left[\left.W_{r}(\beta)\right|_{n}\right]\right)}\right)(x) \\
& \leq \sum_{\substack{\vartheta \in \Sigma^{n} \backslash \mathfrak{W}_{r, n}(\beta) \\
[\vartheta] \subseteq\left[\left.W_{r}(\beta)\right|_{N}\right]}} \varrho \cdot \lambda([\vartheta]) \cdot \sup \{v(y): y \in[\vartheta]\} \\
& \leq \sum_{k=N+1}^{n} \sum_{\substack{\vartheta \in \Sigma^{k} \backslash \mathfrak{W}_{r, k}(\beta) \\
[\vartheta] \subseteq\left[\left.W_{r}(\beta)\right|_{k-1}\right]}} \varrho \cdot \lambda([\vartheta]) \cdot \sup \{v(y): y \in[\vartheta]\} \\
& \leq \sum_{k=N+1}^{n} \sum_{\substack{\vartheta \in \Sigma^{k} \backslash \mathfrak{W}_{r, k}(\beta) \\
[\vartheta] \subseteq\left[\left.W_{r}(\beta)\right|_{k-1}\right]}} \varrho \cdot C_{2} \cdot \lambda([\vartheta]) \cdot \sup \left\{|y-\beta|^{-\alpha}: y \in[\vartheta]\right\} \\
& \leq \sum_{k=N+1}^{n} 2 \cdot \varrho^{2} \cdot C_{2} \cdot\left(\frac{4^{\alpha} \cdot \lambda\left(\left[\left.\vartheta_{r}^{-}(\beta)\right|_{k-1}\right]\right)^{1-\alpha}}{(2-r)^{1+\alpha}}+\frac{4^{\alpha} \cdot \lambda\left(\left[\left.\vartheta \vartheta_{r}^{+}(\beta)\right|_{k-1}\right]\right)^{1-\alpha}}{(2-r)^{1+\alpha}}\right) \\
& \leq \sum_{k=N+1}^{n} \varrho^{2} \cdot C_{2} \cdot 4^{1+\alpha}(2-r)^{-(1+\alpha)-(k-1)(1-\alpha)}
\end{aligned}
$$

This completes the proof.

Remark 7. In the case when one is in the situation of Remark 1, that is when one considers a piecewise $C^{1+\epsilon}$ Markov interval map $T$ : $[0,1] \circlearrowleft$, a similar result to Lemma 4.5 holds true. Specifically, one can show that for a compact interval $[a, b]$ of the open interval $(0,1)$, one has that

$$
\lim _{n \rightarrow \infty} \mathcal{P}^{n}\left(v \cdot \mathbb{1}_{[0,1] \backslash\left[W_{r, n}(\beta)\right]}\right)=\int v \mathrm{~d} \lambda \cdot h_{r},
$$

uniformly on $[a, b]$. (Here $\mathcal{P}$ denotes the Perron-Frobenius operator of $T$.) One approaches this by first showing the results for the end points of $a$ and $b$. This is obtained by a similar arguments to those presented above, however, instead of using Lemma 4.3, one uses the observation that there exists a positive constant $K$ such that

$$
\begin{aligned}
K^{-1} \cdot \min \{a, 1-a\} \cdot\left|g_{n}(0)-g_{n}(1)\right| & \leq\left|g_{n}(a)-g_{n}(t)\right| \\
& \leq K \cdot \max \{a, 1-a\} \cdot\left|g_{n}(0)-g_{n}(1)\right|,
\end{aligned}
$$

where $g_{n}$ denotes an inverse branch of $T^{n}$ and $t=0$ or $t=1$. This follows from an application of the principle of bounded distortion and the chain rule. The 
result stated in (12) will then follow for all $z \in[a, b]$ by monotonicity, and thus the convergence at $z$ only depends on $a$ and $b$, yielding uniform convergence on the interval $[a, b]$.

\subsubsection{Convergence of the $r$-Tail.}

Lemma 4.6. For $r \in[0,1), \alpha \in(0,1), \beta \in[0,1], n \in \mathbb{N}$ and $\eta>0$, we have that

$$
\operatorname{dim}_{\mathcal{H}}\left(\limsup _{n \rightarrow+\infty} A_{n, r, \eta}\right)=0,
$$

where $A_{n, r, \eta}$ is as defined in Definition 4.1 .

Proof. Set $z=T_{r}^{n}(\beta)$ and observe that $z$ is the unique real number in $[0,1]$ with $f_{r,\left.\vartheta_{r}(\beta)\right|_{n}}(z)=\beta$. By the mean value theorem there exists $u \in(0,1)$ such that

$$
\begin{aligned}
\left|\beta-f_{r,\left.\vartheta_{r}(\beta)\right|_{n}}(x)\right| & =\left|f_{r,\left.\vartheta_{r}(\beta)\right|_{n}}(z)-f_{r,\left.\vartheta_{r}(\beta)\right|_{n}}(x)\right| \\
& =|x-z| \cdot\left|f_{r,\left.\vartheta_{r}(\beta)\right|_{n}}^{\prime}(u)\right| \\
& =\left|x-T_{r}^{n}(\beta)\right| \cdot\left|f_{r,\left.\vartheta_{r}(\beta)\right|_{n}}^{\prime}(u)\right| .
\end{aligned}
$$

Further, by construction, we have that $\left|\beta-f_{r,\left.\vartheta_{r}^{ \pm}(\beta)\right|_{n}}(x)\right| \geq\left|\beta-f_{r,\left.\vartheta_{r}(\beta)\right|_{n}}(x)\right|$. This in tandem with (11) and Lemmata 4.2 and 4.3 , yields the following set inclusions.

$$
\begin{aligned}
A_{n, r, \eta} & =\left\{x \in[0,1]: v^{n, r}(x)>\eta\right\} \\
& =\left\{x \in[0,1]: \sum_{\vartheta \in \mathfrak{W}_{r, n}(\beta)}\left|f_{r, \vartheta}^{\prime}(x)\right| \cdot v_{\beta, \alpha} \circ f_{r, \vartheta}>\eta\right\} \\
& =\left\{x \in[0,1]: \sum_{\vartheta \in \mathfrak{W}_{r, n}(\beta)}\left|f_{r, \vartheta}^{\prime}(x)\right| \cdot\left|x-T_{r}^{n}(\beta)\right|^{-\alpha} \cdot\left|f_{r,\left.\vartheta_{r}(\beta)\right|_{n}}^{\prime}(u)\right|^{-\alpha}>\eta\right\} \\
& \subseteq\left\{x \in[0,1]:\left|x-T_{r}^{n}(\beta)\right|<(2-r)^{(1-1 / \alpha) \cdot n} \cdot(3 \cdot \eta \cdot K)^{1 / \alpha}\right\} \\
& =B\left(T_{r}^{n}(\beta),(2-r)^{(1-1 / \alpha) \cdot n} \cdot(3 \cdot \eta \cdot K)^{1 / \alpha}\right)
\end{aligned}
$$

(Here and throughout we denote by $B(y, l)$, the open Euclidean ball centred at $y$ of radius $l$.) Hence, given $\delta>0$, there exists $M=M(\delta) \in \mathbb{N}$ such that

$$
\left\{B\left(T_{r}^{n}(\beta),(2-r)^{(1-1 / \alpha) \cdot n} \cdot(3 \cdot \eta \cdot K)^{1 / \alpha}\right): n \geq M \text { and } n \in \mathbb{N}\right\}
$$

is an open $\delta$-cover of $\limsup _{n \rightarrow+\infty} A_{n, r, \eta}$. Therefore, for $s>0$ and $\delta>0$, letting $\mathcal{H}_{\delta}^{s}$ denote the $\delta$-approximation to the $s$-dimensional Hausdorff measure, we have that

$$
\begin{aligned}
\mathcal{H}_{\delta}^{s} & \left(\limsup _{n \rightarrow+\infty} A_{n, r, \eta}\right) \\
& \leq \sum_{n=M}^{+\infty} \lambda\left(B\left(T_{r}^{n}(\beta),(2-r)^{(1-1 / \alpha) \cdot n} \cdot(3 \cdot \eta \cdot K)^{1 / \alpha}\right)\right)^{s} \\
& \leq \sum_{n=M}^{+\infty}(2-r)^{(1-1 / \alpha) \cdot s \cdot n} \cdot(3 \cdot \eta \cdot K)^{s / \alpha}
\end{aligned}
$$




$$
=\frac{(3 \cdot \eta \cdot K)^{s / \alpha} \cdot(2-r)^{(1-1 / \alpha) \cdot s \cdot M}}{1-(2-r)^{(1-1 / \alpha) \cdot s}} .
$$

Since $\alpha \in(0,1)$, this latter quantity is finite for all $s>0$ and $\delta>0$, and so $\mathcal{H}^{s}\left(\limsup _{n \rightarrow+\infty} A_{n, r, \eta}\right)$ is finite for all $s>0$. This yields that $\operatorname{dim}_{\mathcal{H}}$ $\left(\limsup \sup _{n \rightarrow+\infty} A_{n, r, \eta}\right)=0$ as required. (Here $\mathcal{H}^{s}$ denotes the $s$-dimensional Hausdorff measure.)

\subsection{Auxiliary Results for the Case $r=1$}

4.2.1. Infinite Ergodic Theory Revisited. The transfer operator $\widehat{T}_{1}: \mathcal{L}_{1}^{1}([0,1])$ $\circlearrowleft$ of $T_{1}$ is defined by

$$
\widehat{T}_{1}(f):=\frac{\mathcal{P}_{1}\left(f \cdot h_{1}\right)}{h_{1}} .
$$

Namely, $\widehat{T}_{1}$ is the dual operator of $T_{1}$ with respect to $\mu_{1}$; that is the positive linear operator satisfying

$$
\widehat{T}_{1}(f):=\frac{\mathrm{d} \nu_{1, f} \circ T_{1}^{-1}}{\mathrm{~d} \mu_{1}}, \quad \text { where } \quad \nu_{1, f}(A):=\int \mathbb{1}_{A} \cdot f \mathrm{~d} \mu_{1},
$$

for all Borel sets $A \subset[0,1]$.

Note, the domain of definition of $\widehat{T}_{1}$ can be extended to any well-defined real-valued function.

Let $Y \subset[0,1]$ be such that $\mu_{1}(Y)$ is positive and finite. For each $n \in \mathbb{N}$, define the return time operator $T_{Y}^{(n)}: \mathcal{L}_{1}^{1}([0,1]) \circlearrowleft$ by

$$
T_{Y}^{(n)}(f):=\mathbb{1}_{Y} \cdot \widehat{T}_{1}^{n}\left(\mathbb{1}_{Y} \cdot f\right),
$$

and define the first return time operator $R_{n}: \mathcal{L}_{1}^{1}([0,1]) \circlearrowleft$ by

$$
R_{n}(f):=\mathbb{1}_{Y} \cdot \widehat{T}_{1}^{n}\left(\mathbb{1}_{\left\{y \in Y: \phi_{Y}(y)=n\right\}} \cdot f\right) .
$$

Here $\phi_{Y}(y)$ denotes the first return time of $y \in Y$ given by

$$
\phi_{Y}(y):=\inf \left\{n \in \mathbb{N}: T_{1}^{n}(y) \in Y\right\} .
$$

We let $\mathcal{L}^{\infty}(Y)$ denote the Banach space of equivalence classes $[f]$ of functions, where for each representative $h:[0,1] \rightarrow \mathbb{C}$ of $[f]$, we have that $h$ is a Lebesgue measurable function with

$$
\|h\|_{\mathcal{L}^{\infty}}:=\inf \left\{\|f\|_{\infty}: \lambda\{x: f(x) \neq h(x)\}=0\right\}<+\infty
$$

and with $h$ vanishing on the complement of $Y$. Here $f, g$ belong to the same equivalence class, if and only if, $\|f-g\|_{\mathcal{L}^{\infty}}=0$. Following convention, we will write $f \in \mathcal{L}^{\infty}([0,1])$ to mean a function $f:[0,1] \rightarrow \mathbb{C}$ belonging to an equivalence class of $\mathcal{L}^{\infty}([0,1])$.

Let $\mathcal{B}$, equipped with a norm $\|\cdot\|_{\mathcal{B}}$, be a Banach space of $\mathbb{C}$-valued functions $f \in \mathcal{L}_{1}^{1}([0,1])$ with domain $[0,1]$ that vanish on the complement of $Y$ and which satisfy the following five conditions.

(R1) If $f \in \mathcal{B}$, then $f \in \mathcal{L}^{\infty}([0,1])$ and $R(1)(f) \in \mathcal{B}$, where $R(1):=\sum_{n=1}^{+\infty} R_{n}$. (R2) The inequality $\|f\|_{\mathcal{L}^{\infty}} \leq\|f\|_{\mathcal{B}}$ holds for all $f \in \mathcal{B}$. 
(R3) For all $n \in \mathbb{N}$, the operator $\left.R_{n}\right|_{\mathcal{B}}$ is bounded and linear. Moreover, there exists a constant $C>0$, such that $\left\|R_{n}\right\| \leq C \cdot \mu_{1}\left(\left\{y \in Y: \phi_{Y}(y)=n\right\}\right)$.

(R4) Spectral Gap: The operator $R(1)$ restricted to $\mathcal{B}$ has a simple isolated eigenvalue at 1 .

(R5) Aperiodocity: For $z \in \mathbb{D} \backslash\{1\}$, the value 1 is not in the spectrum of $R(z):=\sum_{n=1}^{+\infty} z^{n} R_{n}: \mathcal{B} \circlearrowleft$. (Here $\mathbb{D}$ denotes the closed unit ball in $\mathbb{C}$.)

Theorem 4.7. ([35, Theorem 2.1]) If conditions (R1) to (R5) are satisfied, then the limit

$$
\lim _{n \rightarrow+\infty} \sup _{f \in \mathcal{B} ;\|f\|_{\mathcal{B}} \leq 1}\left\|\ln (n) \cdot T_{Y}^{(n)}(f)-\int_{Y} f \mathrm{~d} \mu\right\|_{\mathcal{B}},
$$

exists and converges to zero.

In the following proposition, we give an example of when the conditions (R1)-(R5) are satisfied. Analogous results in an $\mathcal{L}^{1}$ setting are abundant in the current literature; the Banach space considered here differs in that it distinguishes functions pointwise and so slight differences arise, which do not change any of the theoretical properties.

Proposition 4.8. Let $Y=[1 / 2,1]$ and let $\mathrm{BV}(Y)$ denote the space of $\mathbb{C}$-valued right-continuous functions with domain $[0,1]$ that vanish on the complement of $Y$ and which are of bounded variation. We define, for all $f \in \mathrm{BV}(Y)$, the norm $\|f\|_{\mathrm{BV}}:=\|f\|_{\infty}+V_{Y}(f)$. The space $\left(\mathrm{BV}(Y),\|\cdot\|_{\mathrm{BV}}\right)$ is a Banach space and satisfies conditions (R1)-(R5).

Proof. See $[13$, p. 74$]$ for the statement that the space $\left(\mathrm{BV}(Y),\|\cdot\|_{\mathrm{BV}}\right)$ is a Banach space. Condition (R1) follows from [38, Proposition 1] together with properties of functions of bounded variation, see [4, Chapter 2] and [13, p. 74 f.]. Condition (R2) follows directly from the definition of the involved norm. Condition (R3) is a direct calculation using properties of functions of bounded variation. By verifying the conditions of [21, Theorem XIV.3] (a generalisations of the results of Doeblin and Fortet [8] and Ionescu-Tulcea and Marinescu [22]) one obtains Condition (R4). Finally, Condition (R5) can be deduced by using similar arguments to presented in [16, Lemma 6.7].

For $k \in \mathbb{N}_{0}$, set

$$
Y_{k}:=T_{1}^{-k}(Y) \backslash \bigcup_{j=0}^{k-1} T_{1}^{-j}(Y)
$$

Indeed, if $Y=[1 / 2,1]$, then $Y_{0}=Y$ and $Y_{k}=[1 /(k+2), 1 /(k+1))$ for $k \geq 1$. For $f:[0,1] \rightarrow \mathbb{C}$ with $\|f\|_{\infty}<\infty$, we let $\widetilde{f}_{k}:=\mathbb{1}_{Y_{k}} \cdot f$ and write $f \in \mathcal{B}([0,1])$, if $f \in \mathcal{L}_{1}^{1}([0,1])$ and $\widehat{T}_{1}^{k}\left(\widetilde{f}_{k}\right) \in \mathcal{B}$ for all $k \in \mathbb{N}_{0}$.

The operator $\widehat{T}_{1}$ can be written in terms of the inverse branches of $T_{1}$, namely

$$
\widehat{T}_{1}(f)(x)=f_{1,0}(x) \cdot f \circ f_{1,1}(x)+f_{1,1}(x) \cdot f \circ f_{1,0}(x) .
$$


This implies, on $[0,1]$, for all $n \in \mathbb{N}$ and integers $j>n$, that $\mathbb{1}_{Y} \cdot \widehat{T}_{1}^{n}\left(\widetilde{f}_{j}\right)=0$ and $\widehat{T}_{1}^{n}\left(\tilde{f}_{n}\right)=\mathbb{1}_{Y} \cdot \widehat{T}_{1}^{n}\left(\tilde{f}_{n}\right)$, and hence, that

$$
\mathbb{1}_{Y} \cdot \widehat{T}_{1}^{n}(f)=\sum_{j=0}^{n} \mathbb{1}_{Y} \cdot \widehat{T}_{1}^{n-j}\left(\mathbb{1}_{Y} \cdot \widehat{T}_{1}^{j}\left(\tilde{f}_{j}\right)\right) .
$$

See [28, p. 11] or [23, Section 3.3.2] for further details on the transfer operator $\widehat{T}_{1}$, the Perron Frobenius operator $\mathcal{P}_{1}$ and the equalities given above.

Theorem 4.9. ([35, Theorem 10.4] and $[24$, Theorem 1.3(i) $])$ Fix $f \in \mathcal{B}([0,1])$ with $\|f\|_{\infty}<+\infty$. If

$$
\sum_{k=0}^{+\infty}\left\|\widehat{T}_{1}^{k}\left(\tilde{f}_{k}\right)\right\|_{\infty}<+\infty
$$

then on $Y$

$$
\lim _{n \rightarrow+\infty} \ln (n) \cdot \widehat{T}_{1}^{n}(f)=\int f \mathrm{~d} \mu_{1} .
$$

Remark 8. If $f \in \mathrm{BV}(0,1)$, then $f / h_{1}$ satisfies the conditions of Theorem 4.9. To see this observe that, by the identity given in (13),

$$
\widehat{T}_{1}^{n}\left(f / h_{1} \cdot \mathbb{1}_{Y_{n}}\right)=\prod_{k=0}^{n-1} f_{1,1} \circ f_{1,0}^{k} \cdot \frac{f \circ f_{1,0}^{n}}{h_{1} \circ f_{1,0}^{n}} \cdot \mathbb{1}_{Y} .
$$

Therefore, since $f, f_{1,0}$ and $f_{1,1}$ are of bounded variation and the composition and product of functions of bounded is again of bounded variation it follows that $\widehat{T}^{n}\left(f / h_{1} \cdot \mathbb{1}_{Y_{n}}\right) \in \mathrm{BV}(Y)$. Moreover, since a function of bounded variation has finite supremum norm, we have that

$$
\sum_{k=0}^{+\infty}\left\|\widehat{T}_{1}^{k}\left(f / h_{1} \cdot \mathbb{1}_{Y_{k}}\right)\right\|_{\infty} \leq \sum_{k=0}^{+\infty}(k+1)^{-2}\|f\|_{\infty}<+\infty .
$$

Theorem 4.10. ([23, Theorem 8] and $\left[24\right.$, Theorem 1.1]) If $f \in \mathcal{L}_{1}^{1}([0,1])$ satisfies

$$
\ln (n) \cdot \widehat{T}_{1}^{n}(f) \rightarrow \int f d \mu_{1}
$$

uniformly on $Y$, then the same convergence holds on any compact subsets of $(0,1]$.

Our next result, Lemma 4.12, is the analogous result of Lemma 4.5 for $r=1$. In the proof of this result the following will play an essential role. For $n \in \mathbb{N}$ and $\beta \in(0,1]$, We recall that $p_{n}=p_{n}(\beta)$ and $q_{n}=q_{n}(\beta)$ are as defined in $(3)$, and define $k(n)=k(n, \beta), m(n)=m(n, \beta)$ and $r(n)=r(n, \beta)$ by

$$
\begin{aligned}
k(n) & :=\max \left\{k \in\{1,2, \ldots, n\}: \vartheta_{1, k}(\beta)=1\right\}, \\
m(n) & :=\operatorname{card}\left\{\ell \in\{1,2, \ldots, n\}: \vartheta_{1, \ell}(\beta)=1\right\} \quad \text { and } \\
r(n) & :=n-k(n) .
\end{aligned}
$$

The following list of properties can be discerned from the given definitions and remarks. 
1. If $k(n)=n$, then $a_{m(n)}=n-k(n-1)$.

2. If $\left(b_{m}\right)_{m \in \mathbb{N}}$ is a sequence of positive real numbers, then, for $n \in \mathbb{N}$, we have that

$$
T_{1}\left(\left[0 ; b_{1}, b_{2}, \ldots, b_{n}\right]\right)= \begin{cases}{\left[0 ; b_{1}-1, b_{2}, \ldots, b_{n}\right]} & \text { if } b_{1}>1, \\ {\left[0 ; b_{2}, \ldots, b_{n}\right]} & \text { otherwise. }\end{cases}
$$

3. The function $f_{1,\left.\vartheta_{1}(\beta)\right|_{n}}$ is a Möbius transformation and for all $x \in[0,1]$, $\lim _{n \rightarrow+\infty} f_{1,\left.\vartheta_{1}(\beta)\right|_{n}}(x)=\beta$.

4. For $n \in \mathbb{N}$, we have that

$$
f_{1,\left.\vartheta_{1}(\beta)\right|_{n}}(0)=\frac{p_{m(n)}}{q_{m(n)}}=\left[0 ; a_{1}, a_{2}, \ldots, a_{m(n)}\right]
$$

and

$$
\begin{aligned}
f_{1,\left.\vartheta_{1}(\beta)\right|_{n}}(1) & =\frac{(r(n)+1) \cdot p_{m(n)}+p_{m(n)-1}}{(r(n)+1) \cdot q_{m(n)}+q_{m(n)-1}} \\
& =\left[0 ; a_{1}, a_{2}, \ldots, a_{m(n)}, r(n)+1\right] .
\end{aligned}
$$

Lemma 4.11. For $n \in \mathbb{N}$ and $\beta \in(0,1]$, we have that

$$
f_{1,\left.\vartheta_{1}(\beta)\right|_{n}}(x)=\frac{\left(r(n) \cdot p_{m(n)}+p_{m(n)-1}\right) \cdot x+p_{m(n)}}{\left(r(n) \cdot q_{m(n)}+q_{m(n)-1}\right) \cdot x+q_{m(n)}},
$$

where $p_{n}=p_{n}(\beta)$ and $q_{n}=q_{n}(\beta)$ are as defined in (3).

Proof. The function $f_{1,\left.\vartheta_{1}(\beta)\right|_{n}}$ is a Möbius transformation and moreover, a Möbius transformation is uniquely determined by its values at three distinct points. Let us consider the case when $\vartheta_{1, n}(\beta)=1$. By definition we have that $r(n)=0$ and so the function on the RHS of (15) becomes

$$
x \mapsto \frac{p_{m(n)-1} \cdot x+p_{m(n)}}{q_{m(n)-1} \cdot x+q_{m(n)}} .
$$

By Property (4) given above,

$$
0 \mapsto \frac{p_{m(n)}}{q_{m(n)}}=f_{\left.\vartheta_{1}(\beta)\right|_{n}}(0) \quad \text { and } \quad 1 \mapsto \frac{p_{m(n)-1}+p_{m(n)}}{q_{m(n)-1}+q_{m(n)}}=f_{\left.\vartheta_{1}(\beta)\right|_{n}}(1) .
$$

Since $f_{1,\left.\vartheta_{1}(\beta)\right|_{n}}$ is a contraction, by Banach's fixed point theorem, there exists a unique $x \in[0,1]$ such that $f_{1,\left.\vartheta_{1}(\beta)\right|_{n}}(x)=x$. By Properties $(1)$ and (2) given above the pre-periodic point

$$
\begin{aligned}
& {\left[0 ; \overline{a_{1}, \ldots, a_{m(n)}}\right]} \\
& \quad:=\left[0 ; a_{1}, \ldots, a_{m(n)}, a_{1}, \ldots, a_{m(n)}, a_{1}, \ldots, a_{m(n)}, \ldots, a_{1}, \ldots, a_{m(n)}, \ldots\right]
\end{aligned}
$$

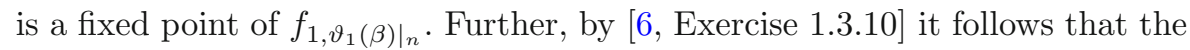
point $\left[0 ; \overline{a_{1}, \ldots, a_{m(n)}}\right]$ is a fixed point of the map given in (16). This completes the proof of the result for when $\vartheta_{n}=1$.

The result for the case when $\left.\vartheta\right|_{n} \neq 1$, follows from the definition of $r(n)$ and the case when $\vartheta_{n}=1$, together with the observation that $f_{1,0}^{n}(x)=$ $x /(1+n \cdot x)$, for $n \in \mathbb{N}$ and all $x \in[0,1]$. 
Lemma 4.12. For $\alpha \in(0,1), \beta \in(0,1]$ of intermediate $\alpha$-type and $v \in \mathfrak{U}_{\beta, \alpha}$, we have that

$$
\lim _{n \rightarrow \infty} \ln (n) \cdot \widehat{T}_{1}^{n}\left(v \cdot \mathbb{1}_{[0,1] \backslash\left[\left.\vartheta_{1}(\beta)\right|_{n}\right]} / h_{1}\right)=\int v \mathrm{~d} \lambda
$$

uniformly on compact subsets of $(0,1)$.

Proof. Let $K$ be a compact subset of $(0,1)$ and let $a, b \in(0,1)$ be such that $K \subseteq[a, b]$. Let $N \in \mathbb{N}$ be fixed. By Proposition 4.8 and Theorems 4.9 and 4.10 together with Remark 8, since the function $v \cdot \mathbb{1}_{[0,1] \backslash\left[\left.\vartheta_{1}(\beta)\right|_{N}\right]}$ is of bounded variation, it follows that, uniformly on $\mathrm{K}$,

$$
\lim _{n \rightarrow \infty} \ln (n) \cdot \widehat{T}_{1}^{n}\left(v \cdot \mathbb{1}_{[0,1] \backslash\left[\left.\vartheta_{1}(\beta)\right|_{N}\right]} / h_{1}\right)=\int v \cdot \mathbb{1}_{[0,1] \backslash\left[\left.\vartheta_{1}(\beta)\right|_{N}\right]} \mathrm{d} \lambda .
$$

Therefore, by linearity and positivity of the operator $\widehat{T}_{1}$, and since $\lim _{k \rightarrow+\infty} \lambda\left(\left[\left.\vartheta_{1}(\beta)\right|_{k}\right]\right)=0$, since the observable $v$ is Lebesgue integrable and since $\beta$ is of intermediate $\alpha$-type, it suffices to show that there exists a positive constant $C$ so that

$$
\lim _{n \rightarrow+\infty} \ln (n) \cdot \widehat{T}_{1}^{n}\left(v_{\beta, \alpha} \cdot \mathbb{1}_{\left[\left.\vartheta_{1}(\beta)\right|_{N}\right] \backslash\left[\left.\vartheta_{1}(\beta)\right|_{n}\right]} / h_{1}\right) \leq C \sum_{k=\widetilde{N}}^{+\infty} \sum_{j=1}^{a_{k}} t_{k, j}^{2 \cdot(\alpha-1)+\epsilon},
$$

for some given $\epsilon \in(0,2 \cdot(1-\alpha))$ and where

1. $t_{n, j}$ is as defined at the end of Sect. 2 and

2. $\quad \widetilde{N}$ is the unique integer so that $a_{1}+\cdots+a_{\widetilde{N}} \leq N \leq a_{1}+\cdots+a_{\widetilde{N}+1}$.

To this end, for each integer $k>1$, we let $\left.\overline{\vartheta_{1}(\beta)}\right|_{k} \in \Sigma^{k}$ be the unique word of length $k$ such that $\left[\left.\vartheta_{1}(\beta)\right|_{k-1}\right]=\left[\left.\vartheta_{1}(\beta)\right|_{k}\right] \cup\left[\left.\overline{\vartheta_{1}(\beta)}\right|_{k}\right]$. By Lemma 4.11 we have that for all $x \in K$

1. $\left|f_{\vartheta_{1}(\beta)}^{\prime}(x)\right| \leq a^{-2} \cdot\left((r(k)+1) \cdot q_{m(k)}+q_{m(k)-1}\right)^{-2}$,

2. if $r(k)+1 \neq a_{m(k)}$, then

$$
\begin{aligned}
\mid \beta & -f_{\left.\overline{\vartheta_{1}(\beta)}\right|_{k}}(x) \mid \\
& \geq\left|\frac{(r(k)+2) \cdot p_{m(k)}+p_{m(k)-1}}{(r(k)+2) \cdot q_{m(k)}+q_{m(k)-1}}-\frac{(r(k)+1) \cdot p_{m(k)}+p_{m(k)-1}}{(r(k)+1) \cdot q_{m(k)}+q_{m(k)-1}}\right| \\
& \geq \frac{1}{2 \cdot\left((r(k)+1) \cdot q_{m(k)}+q_{m(k)-1}\right)^{2}},
\end{aligned}
$$

3. if $r(k)+1=a_{m(k)}$, letting

$$
z_{k}= \begin{cases}b & \text { if } m(k) \text { is even } \\ a & \text { if } m(k) \text { is odd }\end{cases}
$$


then

$$
\begin{aligned}
\mid \beta & -f_{\left.\overline{\vartheta_{1}(\beta)}\right|_{k}}(x) \mid \\
& \geq\left|\frac{(r(k)+1) \cdot p_{m(k)}+p_{m(k)-1}}{(r(k)+1) \cdot q_{m(k)}+q_{m(k)-1}}-\frac{\left(r(k) \cdot p_{m(k)}+p_{m(k)-1}\right) \cdot x+p_{m(k)}}{\left(r(k) \cdot q_{m(k)}+q_{m(k)-1}\right) \cdot x+q_{m(k)}}\right| \\
& \geq \frac{1-z_{k}}{\left((r(k)+1) \cdot q_{m(k)}+q_{m(k)-1}\right)^{2}} .
\end{aligned}
$$

Since a constant function is of bounded variation, we have by Proposition 4.8 and Theorems 4.9 and 4.10 together with Remark 8, that there exists a positive constant $C^{\prime}$, so that

$$
\widehat{T}_{1}^{k}\left(1 / h_{1}\right)(x) \leq \frac{C^{\prime}}{\ln (k+1)},
$$

for all $k \in \mathbb{N}$ and $x \in K$. Noting that $t_{m(k), r(k)+1}=(r(k)+1) \cdot q_{m(k)}+q_{m(k)-1}$ and, letting $\epsilon$ be such that

$$
\sum_{n=1}^{+\infty} \sum_{j=1}^{a_{n}} t_{n, j}^{-2 \cdot(1-\alpha)+\epsilon}<+\infty
$$

we have that

$$
\begin{aligned}
\lim _{n \rightarrow+\infty} & \ln (n) \cdot \widehat{T}_{1}^{n}\left(v_{\beta, \alpha} \cdot \mathbb{1}_{\left[\left.\vartheta_{1}(\beta)\right|_{N}\right] \backslash\left[\left.\vartheta_{1}(\beta)\right|_{n}\right]} / h_{1}\right) \\
= & \lim _{n \rightarrow+\infty} \ln (n) \sum_{k=N+1}^{n-1} \widehat{T}_{1}^{n-k}\left(\widehat{T}_{1}^{k}\left(v_{\beta, \alpha} \cdot \mathbb{1}_{\left[\left.\vartheta_{1}(\beta)\right|_{k}\right]} / h_{1}\right)\right) \\
\leq & \lim _{n \rightarrow+\infty} \frac{C^{\prime}}{2 \cdot a^{2} \cdot\left(1-z_{k}\right)} \\
& \cdot \sum_{k=N+1}^{n-1} \frac{\ln (n)}{\ln (n-k+1)} \frac{1}{\left((r(k)+1) \cdot q_{m(k)}+q_{m(k)-1}\right)^{2 \cdot(1-\alpha)}} \\
\leq & \lim _{n \rightarrow+\infty} \frac{C^{\prime}}{2 \cdot a^{2} \cdot\left(1-z_{k}\right)} \\
& \cdot \sum_{k=N+1}^{\lfloor n / 2\rfloor} \frac{\ln (n)}{\ln (n / 2)} \frac{1}{\left((r(k)+1) \cdot q_{m(k)}+q_{m(k)-1}\right)^{2 \cdot(1-\alpha)-\epsilon}} \\
& +\lim _{n \rightarrow+\infty} \frac{C^{\prime}}{2 \cdot a^{2} \cdot\left(1-z_{k}\right)} \\
& \cdot \sum_{k=\lfloor n / 2\rfloor+1}^{n-1} \frac{2 \cdot \ln (n)}{n^{\epsilon}} \frac{1}{\left((r(k)+1) \cdot q_{m(k)}+q_{m(k)-1}\right)^{2 \cdot(1-\alpha)-\epsilon}}
\end{aligned}
$$




$$
\begin{aligned}
& \leq \frac{C^{\prime}}{a^{2} \cdot\left(1-z_{k}\right)} \sum_{k=N+1}^{+\infty} \frac{1}{\left((r(k)+1) \cdot q_{m(k)}+q_{m(k)-1}\right)^{2 \cdot(1-\alpha)-\epsilon}} \\
& \leq \frac{C^{\prime}}{a^{2} \cdot\left(1-z_{k}\right)} \sum_{k=\widetilde{N}}^{+\infty} \sum_{j=1}^{a_{k}} t_{k, j}^{2 \cdot(\alpha-1)+\epsilon} .
\end{aligned}
$$

This completes the proof.

4.2.2. Convergence of the 1-Tail. The aim of this section is to provide an analogous result (Lemma 4.13) for $r=1$ of Lemma 4.6. The idea behind the proofs of Lemmata 4.6 and 4.13 are similar, however, in the case that $r=1$, several technical difficulties arise and thus need to be taken care off.

Lemma 4.13. For $\alpha \in(0,1), \beta \in[0,1]$ irrational, $n \in \mathbb{N}$ and $\eta>0$, we have that

$$
\operatorname{dim}_{\mathcal{H}}\left(\limsup _{n \rightarrow+\infty} A_{n, 1, \eta}\right)=0
$$

Proof. It is sufficient to prove, for all $k \in \mathbb{N}, \eta>0$ and $\epsilon \in\left(0,(2 k(k+1))^{-1}\right)$, that

$$
\operatorname{dim}_{\mathcal{H}}\left(\limsup _{n \rightarrow+\infty} A_{n, 1, \eta} \cap(1 /(k+1)+\epsilon, 1 / k-\epsilon)\right)=0 .
$$

To this end, for $n \in \mathbb{N}$, set $z=z(n):=T_{1}^{n}(\beta)$ and observe that $z$ is the unique real number in $[0,1]$ such that $f_{1,\left.\vartheta_{1}(\beta)\right|_{n}}(z)=\beta$. If $z \in(1 /(k+1), 1 / k)$, then, for all $x \in(1 /(k+1)+\epsilon, 1 / k-\epsilon)$, by the mean value theorem and Lemma 4.11, there exists $u \in(1 /(k+1), 1 / k)$ such that

$$
\begin{aligned}
\left|\beta-f_{1,\left.\vartheta_{1}(\beta)\right|_{n}}(x)\right| & =\left|f_{1,\left.\vartheta_{1}(\beta)\right|_{n}}(z)-f_{1,\left.\vartheta_{1}(\beta)\right|_{n}}(x)\right| \\
& =|x-z| \cdot\left|f_{1,\left.\vartheta_{1}(\beta)\right|_{n}}^{\prime}(u)\right| \\
& =\left|x-T_{1}^{n}(\beta)\right| \cdot\left|(r(n) u+1) q_{m(n)}+q_{m(n)-1} u\right|^{-2} \\
& \geq k^{2} \cdot\left|x-T_{1}^{n}(\beta)\right| \cdot\left|(r(n)+k) q_{m(n)}+q_{m(n)-1}\right|^{-2} .
\end{aligned}
$$

If $z \notin(1 /(k+1), 1 / k)$, then, for all $x \in(1 /(k+1)+\epsilon, 1 / k-\epsilon)$, since $f_{1,\left.\vartheta_{1}(\beta)\right|_{n}}$ is order preserving or order reversing, we have that

$$
\begin{aligned}
& \left|\beta-f_{1,\left.\vartheta_{1}(\beta)\right|_{n}}(x)\right|=\left|f_{1,\left.\vartheta_{1}(\beta)\right|_{n}}(z)-f_{1,\left.\vartheta_{1}(\beta)\right|_{n}}(x)\right| \\
& \quad \geq \min \left\{\left|f_{1,\left.\vartheta_{1}(\beta)\right|_{n}}(1 / k)-f_{1,\left.\vartheta_{1}(\beta)\right|_{n}}(x)\right|,\left|f_{1,\left.\vartheta_{1}(\beta)\right|_{n}}(1 /(k+1))-f_{1,\left.\vartheta_{1}(\beta)\right|_{n}}(x)\right|\right\}
\end{aligned}
$$

and so by the mean value theorem and Lemma 4.11, there exists $u \in(1 /(k+$ 1), $1 / k$ ) such that

$$
\begin{aligned}
\left|\beta-f_{1,\left.\vartheta_{1}(\beta)\right|_{n}}(x)\right| & \geq \epsilon \cdot\left|f_{1,\left.\vartheta_{1}(\beta)\right|_{n}}^{\prime}(u)\right| \\
& =\epsilon \cdot\left|(r(n) \cdot u+1) \cdot q_{m(n)}+q_{m(n)-1} \cdot u\right|^{-2} \\
& \geq \epsilon \cdot k^{2} \cdot\left|(r(n)+k) \cdot q_{m(n)}+q_{m(n)-1}\right|^{-2} .
\end{aligned}
$$


Hence, for $x \in(1 /(k+1)+\epsilon, 1 / k-\epsilon)$, we have that

$$
\begin{aligned}
& \ln (n) \cdot v^{n, 1}(x) \\
& =\frac{\ln (n)}{\left((r(n) \cdot x+1) \cdot q_{m(n)}+q_{m(n)-1} \cdot x\right)^{2}} \frac{1}{\left|\beta-f_{1,\left.\vartheta_{1}(\beta)\right|_{n}}(x)\right|^{\alpha}} \\
& \quad \leq\left\{\begin{array}{l}
\frac{(k+1)^{2} \cdot \ln (n)}{\left|T_{1}^{n}(\beta)-x\right|^{\alpha} \cdot k^{2 \cdot \alpha} \cdot\left((r(n)+k) \cdot q_{m(n)}+q_{m(n)-1}\right)^{2 \cdot(1-\alpha)}} \\
\frac{(k+1)^{2} \cdot \ln (n)}{\text { if } T_{1}^{n}(\beta) \in(1 /(k+1), 1 / k),} \\
\frac{\text { if } T_{1}^{n}(\beta) \notin(1 /(k+1), 1 / k) .}{\epsilon^{\alpha} \cdot k^{2 \cdot \alpha} \cdot\left((r(n)+k) \cdot q_{m(n)}+q_{m(n)-1}\right)^{2 \cdot(1-\alpha)}}
\end{array}\right.
\end{aligned}
$$

Since,

$$
\begin{aligned}
& \lim _{n \rightarrow+\infty} \frac{(k+1)^{2} \cdot \ln (n)}{\epsilon^{\alpha} \cdot k^{2 \cdot \alpha} \cdot\left((r(n)+k) \cdot q_{m(n)}+q_{m(n)-1}\right)^{2 \cdot(1-\alpha)}} \\
& \quad \leq \lim _{n \rightarrow+\infty} \frac{(k+1)^{2} \cdot \ln \left((r(n)+k) \cdot q_{m(n)}+q_{m(n)-1}\right)}{\epsilon^{\alpha} \cdot k^{2 \cdot \alpha} \cdot\left((r(n)+k) \cdot q_{m(n)}+q_{m(n)-1}\right)^{2 \cdot(1-\alpha)}}=0,
\end{aligned}
$$

there exists $M \in \mathbb{N}$ such that, for all $x \in(1 /(k+1)+\epsilon, 1 / k-\epsilon)$ and $n \geq M$, if $T_{1}^{n}(\beta) \notin(1 /(k+1), 1 / k)$, then $\ln (n) \cdot v_{n, 1}(x)<\eta$. Therefore, for all $n \geq M$, if $T_{1}^{n}(\beta) \notin(1 /(k+1), 1 / k)$, then

$$
A_{n, 1, \eta} \cap(1 /(k+1)+\epsilon, 1 / k-\epsilon)=\emptyset ;
$$

otherwise, if $T_{1}^{n}(\beta) \in(1 /(k+1), 1 / k)$, then

$$
\begin{aligned}
A_{n, 1, \eta} & \cap(1 /(k+1)+\epsilon, 1 / k-\epsilon) \\
= & \left\{x \in(1 /(k+1)+\epsilon, 1 / k-\epsilon): \ln (n) \cdot v^{n, 1}(x) \geq \eta\right\} \\
\subseteq & \{x \in(1 /(k+1)+\epsilon, 1 / k-\epsilon): \\
& \left.\frac{(k+1)^{2} \cdot \ln (n)}{\left|T_{1}^{n}(\beta)-x\right|^{\alpha} \cdot k^{2 \cdot \alpha} \cdot\left((r(n)+k) \cdot q_{m(n)}+q_{m(n)-1}\right)^{2 \cdot(1-\alpha)}} \geq \eta\right\} \\
\subseteq & B\left(T_{1}^{n}(\beta), \frac{(k+1)^{2 / \alpha} \cdot \ln (n)^{1 / \alpha}}{\eta^{1 / \alpha} \cdot k^{2} \cdot\left((r(n)+k) \cdot q_{m(n)}+q_{m(n)-1}\right)^{2 \cdot(1 / \alpha-1)}}\right) \\
& \cap(1 /(k+1)+\epsilon, 1 / k-\epsilon) .
\end{aligned}
$$


Hence, given $\delta>0$, there exists a natural number $K=K(\delta) \geq M$ such that

$$
\begin{aligned}
& \left\{B\left(T_{1}^{n}(\beta), \frac{(k+1)^{2 / \alpha} \cdot \ln (n)^{1 / \alpha}}{\eta^{1 / \alpha} \cdot k^{2} \cdot\left((r(n)+k) \cdot q_{m(n)}+q_{m(n)-1}\right)^{2 \cdot(1 / \alpha-1)}}\right):\right. \\
& \left.n \geq K \text { and } \exists l \in \mathbb{N} \text { so that } n=-k+\sum_{i=1}^{l} a_{i}\right\}
\end{aligned}
$$

is an open $\delta$-cover of $\limsup _{n \rightarrow+\infty} A_{n, 1, \eta} \cap(1 /(k+1)+\epsilon, 1 / k-\epsilon)$. Therefore, for $s>0$ and $\delta>0$, letting $\mathcal{H}_{\delta}^{s}$ denote the $\delta$-approximation to the $s$-dimensional Hausdorff measure, we have that

$$
\begin{aligned}
\mathcal{H}_{\delta}^{s} & \left(\limsup _{n \rightarrow+\infty} A_{\eta, n} \cap(1 /(k+1)+\epsilon, 1 / k-\epsilon)\right) \\
& \leq \sum_{n=M}^{+\infty} \lambda\left(B\left(T_{1}^{n}(\beta), \frac{2^{2 \cdot(1 / \alpha-1)} \cdot(k+1)^{2 / \alpha} \cdot \ln (n)^{1 / \alpha}}{\eta^{1 / \alpha} \cdot k^{2} \cdot\left((r(n)+k+1) \cdot q_{m(n)}+q_{m(n)-1}\right)^{2 \cdot(1 / \alpha-1)}}\right)\right. \\
& \cap(1 /(k+1)+\epsilon, 1 / k-\epsilon)) \\
& \leq \frac{2^{s+2 \cdot(1 / \alpha-1)} \cdot(k+1)^{2 \cdot s / \alpha}}{\eta^{s / \alpha} \cdot k^{2 \cdot s}} \sum_{m=m(K)}^{+\infty} \frac{\ln \left(\sum_{\ell=1}^{m+1} a_{\ell}\right)^{s / \alpha}}{\left(q_{m+1}\right)^{2 \cdot s \cdot(1 / \alpha-1)}} \\
& \leq \frac{2^{s+2 \cdot(1 / \alpha-1)} \cdot(k+1)^{2 \cdot s / \alpha}}{\eta^{s / \alpha} \cdot k^{2 \cdot s}} \sum_{m=m(K)}^{+\infty} \frac{\ln \left(q_{m+1}\right)^{s / \alpha}}{\left(q_{m+1}\right)^{2 \cdot s \cdot(1 / \alpha-1)}} .
\end{aligned}
$$

(In the above we have used that if $y \in[1 /(\ell+2), 1 /(\ell+1)]$, for some $\ell \in \mathbb{N}$, then $T_{1}(y) \in[1 /(\ell+1), 1 / \ell]$.) This latter infinite sum is finite for all $s>0$ and $\delta>0$ since, by the recursive definition of $q_{n}$, we have that $q_{n}$ grows at least at an exponential rate as $n \rightarrow+\infty$. Thus $\mathcal{H}^{s}\left(\lim \sup _{n \rightarrow+\infty} A_{n, 1, \eta}\right)$ is finite for all $s>0$. This yields that $\operatorname{dim}_{\mathcal{H}}\left(\limsup _{n \rightarrow+\infty} A_{n, 1, \eta}\right)=0$ as required. (Here $\mathcal{H}^{s}$ denotes the $s$-dimensional Hausdorff measure.)

\section{Proof of Main Results}

\subsection{Proof of Theorem 3.1}

Proof of Theorem 3.1. By linearity of the Perron-Frobenius operator we have that

$$
\mathcal{P}_{r}^{n}(v)=\mathcal{P}_{r}^{n}\left(v \cdot \mathbb{1}_{[0,1] \backslash\left[W_{r, n}(\beta)\right]}\right)+\mathcal{P}_{r}^{n}\left(v \cdot \mathbb{1}_{\left[W_{r, n}(\beta)\right]}\right)
$$

where $\left[W_{r, n}(\beta)\right]$ is as defined in (8). Further, by Lemma 4.5 we have that

$$
\lim _{n \rightarrow+\infty} \mathcal{P}_{r}^{n}\left(v \cdot \mathbb{1}_{[0,1] \backslash\left[W_{r, n}(\beta)\right]}\right)=\int v \mathrm{~d} \lambda \cdot h_{r}
$$


uniformly on $[0,1]$. By the facts that $v$ is non-negative and $\mathcal{P}_{r}$ is a positive operator, we have that

$$
0 \leq \lim _{n \rightarrow \infty} \mathcal{P}_{r}^{n}\left(v \cdot \mathbb{1}_{\left[W_{r, n}(\beta)\right]}\right) \leq \lim _{n \rightarrow \infty} \mathcal{P}_{r}^{n}\left(v^{n, r}\right),
$$

where $v^{n, r}$ is as defined in (9). By Lemma 4.6, this latter limit is equal to zero outside a set of Hausdorff dimension zero.

All that remains to show is that if $\beta \in[0,1]$ is pre-periodic with respect to $T_{r}$ and has period length strictly greater than one, then on $\omega_{r}(\beta)$ we have that

$$
\liminf _{n \rightarrow+\infty} \mathcal{P}_{r}^{n}(v)=\int v \mathrm{~d} \lambda \cdot h_{r} \quad \text { and } \quad \limsup _{n \rightarrow+\infty} \mathcal{P}_{r}^{n}(v) ;=+\infty ;
$$

and in the case that $\beta \in[0,1]$ is pre-periodic with respect to $T_{r}$ and has period length equal to one then on the singleton $\omega_{r}(\beta)$ we have that the limit in (4) is equal to $+\infty$.

By linearity of $\mathcal{P}_{r}^{n}$ and Lemma 4.5 , it suffices to show, if $\beta \in[0,1]$ is preperiodic with respect to $T_{r}$ and has period length strictly greater than one, then on $\omega_{r}(\beta)$

$$
\liminf _{n \rightarrow+\infty} v^{n, r}=0 \text { and } \quad \limsup _{n \rightarrow+\infty} v^{n, r}=+\infty ;
$$

and in the case that $\beta \in[0,1]$ is pre-periodic with respect to $T_{r}$ and has period length equal to one, then on the singleton $\omega_{r}(\beta)$

$$
\lim _{n \rightarrow+\infty} v^{n, r}=+\infty \text {. }
$$

Indeed if $\beta$ is pre-periodic with respect to $T_{r}$ and has period length $m \geq 1$, then letting $n \in \mathbb{N}_{0}$, be the minimal integer so that $T_{r}^{n+k}(\beta)=T_{r}^{n+k+m}(\beta)$, for all $k \in \mathbb{N}_{0}$, we have that

$$
f_{r,\left(\vartheta_{r, n+j+1}(\beta), \ldots, \vartheta_{r, n+j+m}(\beta)\right)}\left(T_{r}^{n+j}(\beta)\right)=T_{r}^{n+j}(\beta),
$$

for all $j \in\{0,1, \ldots, m-1\}$. Further, $\omega_{r}(\beta)=\left\{T_{r}^{n}(\beta), \ldots, T_{r}^{n+m-1}(\beta)\right\}$, and hence, for $j \in\{0,1, \ldots, m-1\}$, it follows that $v^{n+j+k \cdot m, r}\left(T_{r}^{n+j}(\beta)\right)=+\infty$, for all $k \in \mathbb{N}_{0}$. To complete the proof we will show, for $m>1$ and $i, j \in$ $\{0,1, \ldots, m-1\}$ with $i \neq j$, that

$$
\lim _{k \rightarrow+\infty} v^{n+j+k \cdot m, r}\left(T_{r}^{n+i}(\beta)\right)=0 .
$$

For this set $L:=\min \left\{\mid T_{r}^{n+j}(\beta)-T_{r}^{n+i}(\beta): i, j \in\{0,1, \ldots, m-1\}\right.$ and $\left.i \neq j\right\}$. By (11) and Lemmata 4.2 and 4.3 , there exists a positive constant $\varrho \in \mathbb{R}$ such that the following chain of inequalities hold.

$$
\begin{aligned}
\lim _{k \rightarrow+\infty} v^{n+j+k \cdot m, r}\left(T_{r}^{n+i}(\beta)\right) \\
=\lim _{k \rightarrow+\infty} \sum_{\vartheta \in \mathfrak{W}_{r, n+j+k \cdot m}(\beta)}\left|f_{r, \vartheta}^{\prime}\left(T_{r}^{n+i}(\beta)\right)\right| \cdot\left|\beta-f_{r, \vartheta}\left(T_{r}^{n+i}(\beta)\right)\right|^{-\alpha} \\
\leq \lim _{k \rightarrow+\infty} 3 \cdot \varrho \cdot\left|f_{r,\left.\vartheta_{1}(\beta)\right|_{n+j+k \cdot m} ^{\prime}}^{\prime}\left(T_{r}^{n+i}(\beta)\right)\right| \\
\quad \cdot\left|\beta-f_{r,\left.\vartheta_{1}(\beta)\right|_{n+j+k \cdot m}}\left(T_{r}^{n+i}(\beta)\right)\right|^{-\alpha}
\end{aligned}
$$




$$
\begin{aligned}
\leq & \lim _{k \rightarrow+\infty} 3 \cdot \varrho \cdot\left|f_{r,\left.\vartheta_{1}(\beta)\right|_{n+j+k \cdot m}}^{\prime}\left(T_{r}^{n+i}(\beta)\right)\right| \\
& \cdot\left|f_{r,\left.\vartheta_{1}(\beta)\right|_{n+j+k \cdot m}}\left(T_{r}^{n+j+k \cdot m}(\beta)\right)-f_{r,\left.\vartheta_{1}(\beta)\right|_{n+j+k \cdot m}}\left(T_{r}^{n+i}(\beta)\right)\right|^{-\alpha} \\
\leq & \lim _{k \rightarrow+\infty} 3 \cdot \varrho^{1+\alpha} \cdot\left|f_{r,\left.\vartheta_{1}(\beta)\right|_{n+j+k \cdot m}}^{\prime}\left(T_{r}^{n+i}(\beta)\right)\right|^{1-\alpha} \\
& \cdot\left|T_{r}^{n+j+k \cdot m}(\beta)-T_{r}^{n+i}(\beta)\right|^{-\alpha} \\
= & 3 \cdot \varrho^{1+\alpha} \cdot\left|T_{r}^{n+j}(\beta)-T_{r}^{n+i}(\beta)\right|^{-\alpha} \lim _{k \rightarrow \infty}(2-r)^{(\alpha-1) \cdot(n+j+k \cdot m)} \\
= & 3 \cdot \varrho^{1+\alpha} \cdot L \lim _{k \rightarrow \infty}(2-r)^{(\alpha-1) \cdot(n+j+k \cdot m)}=0 .
\end{aligned}
$$

This completes the proof.

\subsection{Proof of Theorems 3.2 and 3.3}

5.2.1. Proof of Theorem 3.2. We divide the proof of Theorem 3.2 into two cases; the first case is when $\beta$ is a rational number and the second case is when $\beta$ is an irrational of intermediate $\alpha$-type. We emphasise that when $\beta$ is an irrational of intermediate $\alpha$-type, then the method of proof of Theorem 3.2 is the same as Theorem 3.1, whereas in the case that $\beta$ is a rational, this method is no longer applicable.

Proof of Theorem 3.2 for $\beta$ Rational. Let $\alpha \in(0,1), \beta \in(0,1]$ be a rational number and $v \in \mathfrak{U}_{\beta, \alpha}$. As $\beta$ is a rational number, there exists a minimal $n \in \mathbb{N}$ such that $T^{n}(\beta)=0$, let $n$ be fixed as such. Further, we have that $\omega_{1}(\beta)=\{0\}$. We will first prove the result for $\beta \neq 1$. By definition of the Farey map, there exist exactly two finite words $\eta, \eta^{\prime} \in \Sigma^{n}$ such that

(a) $f_{1, \eta}(0)=\beta=f_{1, \eta^{\prime}}(0)$,

(b) $f_{1, \eta}(x)<\beta<f_{1, \eta^{\prime}}(x)$, for all $x \in(0,1]$, and

(c) $f_{1, \xi}(x) \neq \beta$, for all words $\xi \in \Sigma^{n} \backslash\left\{\eta, \eta^{\prime}\right\}$ and all $x \in[0,1]$.

By definition, we have, for $k \in \mathbb{N}$, that

$$
\mathcal{P}_{1}^{k}(v)(x)=\sum_{\xi \in \Sigma^{k}}\left|f_{1, \xi}^{\prime}\right| \cdot v \circ f_{1, \xi} .
$$

Hence, by linearity of the operator $\mathcal{P}_{1}$, we have, for all natural numbers $k>n$, that

$$
\begin{aligned}
\mathcal{P}_{1}^{k}(v) & =\mathcal{P}_{1}^{k-n}\left(\mathcal{P}_{1}^{n}(v)\right) \\
& =\mathcal{P}_{1}^{k-n}\left(\mathcal{P}_{1}^{n}\left(v \cdot \mathbb{1}_{[0,1] \backslash[\eta] \cap[0,1] \backslash\left[\eta^{\prime}\right]}\right)\right)+\mathcal{P}_{1}^{k-n}\left(\mathcal{P}_{1}^{n}\left(v \cdot \mathbb{1}_{[\eta] \cup\left[\eta^{\prime}\right]}\right)\right) \\
& =\mathcal{P}_{1}^{k-n}\left(\sum_{\xi \in \Sigma^{k} \backslash\left\{\eta, \eta^{\prime}\right\}}\left|f_{1, \xi}^{\prime}\right| \cdot v \circ f_{1, \xi}\right)+\mathcal{P}_{1}^{k-n}\left(\mathcal{P}_{1}^{n}\left(v \cdot \mathbb{1}_{[\eta] \cup\left[\eta^{\prime}\right]}\right)\right) .
\end{aligned}
$$

If $\xi \in\{0,1\}^{n-1} \backslash\left\{\eta, \eta^{\prime}\right\}$, then since $\beta \notin f_{1, \xi}([0,1])$, since the functions $f_{1, \xi}, f_{1, \xi}^{\prime}$, $1 / h_{1}$ are all of bounded variation, since $v \in \mathfrak{U}_{\beta, \alpha}$ and since $[\xi]$ is a compact interval bounded away from $\beta$, by the fact that the composition, sum, difference and product of two functions of bounded variation is again of bounded 
variation (see [4, Chapter 2]), it follows that the function

$$
[0,1] \ni x \mapsto \frac{1}{h_{1}(x)} \sum_{\xi \in \Sigma^{k} \backslash\left\{\eta, \eta^{\prime}\right\}}\left|f_{1, \xi}^{\prime}(x)\right| \cdot v \circ f_{1, \xi}(x)
$$

is of bounded variation. Hence, by Proposition 4.8 and Theorems 4.9 and 4.10 together with Remark 8, we have that

$$
\begin{aligned}
\lim _{k \rightarrow \infty} \ln (k) \cdot \mathcal{P}_{1}^{k}\left(v \cdot \mathbb{1}_{[0,1] \backslash[\eta] \cap[0,1] \backslash\left[\eta^{\prime}\right]}\right) & =\int \mathcal{P}_{1}^{n}\left(v \cdot \mathbb{1}_{[0,1] \backslash[\eta] \cap[0,1] \backslash\left[\eta^{\prime}\right]}\right) \mathrm{d} \lambda \cdot h_{1} \\
& =\int v \cdot \mathbb{1}_{[0,1] \backslash[\eta] \cap[0,1] \backslash\left[\eta^{\prime}\right]} \mathrm{d} \lambda \cdot h_{1} .
\end{aligned}
$$

Therefore, to complete the proof we need to show that

$$
\lim _{k \rightarrow+\infty} \ln (k) \cdot \mathcal{P}_{1}^{k}\left(v \cdot \mathbb{1}_{[\eta] \cup\left[\eta^{\prime}\right]}\right)=\int v \cdot \mathbb{1}_{[\eta] \cup\left[\eta^{\prime}\right]} \mathrm{d} \lambda \cdot h_{1} .
$$

To this end let $m>n$ be a fixed natural number satisfying

$$
\lambda([\xi]) \leq \min \{|a-\beta|,|b-\beta|\}
$$

for all $\xi \in \Sigma^{m}$, where $U=(a, b)$ is the open connected set such that $C_{1} v_{\beta, \alpha} \leq$ $v \leq C_{2} v_{\beta, \alpha}$ on $U$, for some constants $C_{1}, C_{2}$. Let $\nu, \nu^{\prime} \in \Sigma^{m}$ be the unique words satisfying $[\nu] \cap\left[\nu^{\prime}\right]=\{\beta\},[\nu] \subset[\eta]$ and $\left[\nu^{\prime}\right] \subset\left[\eta^{\prime}\right]$. Indeed, we necessarily have that $f_{1, \nu}(0)=\beta=f_{1, \nu^{\prime}}(0)$. Using identical arguments to those above, we can conclude that

$$
\lim _{k \rightarrow+\infty} \ln (k) \cdot \mathcal{P}_{1}^{k}\left(v \cdot \mathbb{1}_{[\eta] \backslash[\nu] \cup\left[\eta^{\prime}\right] \backslash\left[\nu^{\prime}\right]}\right)=\int v \cdot \mathbb{1}_{[\eta] \backslash[\nu] \cup\left[\eta^{\prime}\right] \backslash\left[\nu^{\prime}\right]} \mathrm{d} \lambda \cdot h_{1} .
$$

Moreover, by positivity of the operator $\mathcal{P}_{1}$ we have that

$$
C_{1} \mathcal{P}_{1}^{k}\left(v_{\beta, \alpha} \cdot \mathbb{1}_{[\nu] \cup\left[\nu^{\prime}\right]}\right) \leq \mathcal{P}_{1}^{k}\left(v \cdot \mathbb{1}_{[\nu] \cup\left[\nu^{\prime}\right]}\right) \leq C_{2} \mathcal{P}_{1}^{k}\left(v_{\beta, \alpha} \cdot \mathbb{1}_{[\nu] \cup\left[\nu^{\prime}\right]}\right) .
$$

We claim (and will shortly prove) that

$$
\lim _{k \rightarrow+\infty} \ln (k) \cdot \mathcal{P}_{1}^{k}\left(v_{\beta, \alpha} \cdot \mathbb{1}_{[\nu] \cup\left[\nu^{\prime}\right]}\right)=\int v_{\beta, \alpha} \cdot \mathbb{1}_{[\nu] \cup\left[\nu^{\prime}\right]} \mathrm{d} \lambda \cdot h_{1} .
$$

Assuming this, we may conclude, for all $m \in \mathbb{N}$, that

$$
\begin{aligned}
& \liminf _{k \rightarrow+\infty} \ln (k) \cdot \mathcal{P}_{1}^{k}(v) \\
& \quad \geq C_{1} \int v_{\beta, \alpha} \cdot \mathbb{1}_{[\nu] \cup\left[\nu^{\prime}\right]} \mathrm{d} \lambda \cdot h_{1}+\int v \cdot \mathbb{1}_{[0,1] \backslash[\nu] \cap[0,1] \backslash\left[\nu^{\prime}\right]} \mathrm{d} \lambda \cdot h_{1}
\end{aligned}
$$

and

$$
\begin{aligned}
& \limsup _{k \rightarrow+\infty} \ln (k) \cdot \mathcal{P}_{1}^{k}(v) \\
& \quad \leq C_{2} \int v_{\beta, \alpha} \cdot \mathbb{1}_{[\nu] \cup\left[\nu^{\prime}\right]} \mathrm{d} \lambda \cdot h_{1}+\int v \cdot \mathbb{1}_{[0,1] \backslash[\nu] \cap[0,1] \backslash\left[\nu^{\prime}\right]} \mathrm{d} \lambda \cdot h_{1} .
\end{aligned}
$$

(Note that the words $\nu, \nu^{\prime}$ are dependent on $m$.) Since the LHS of (18) and (19) are independent of $m$ and since $\lambda(\nu), \lambda\left(\nu^{\prime}\right)$ both converge to zero as $n \rightarrow+\infty$, the result follows.

We now prove the equality given in (17). By Proposition 4.8 and Theorems 4.9 and 4.10 together with Remark 8 it is sufficient to show that 
$[0,1] \ni x \mapsto \widehat{T}_{1}^{m}\left(v_{\beta, \alpha} \cdot \mathbb{1}_{[\nu] \cup\left[\nu^{\prime}\right]} / h_{1}\right)(x)$ is of bounded variation. In order to show this, recall that $f_{1, \nu}$ and $f_{1, \nu^{\prime}}$ are Möbius transformations and observe that

$$
\widehat{T}_{1}^{m}\left(v_{\beta, \alpha} \cdot \mathbb{1}_{[\nu] \cup\left[\nu^{\prime}\right]} / h_{1}\right)(x)=\sum_{i=1}^{2} \frac{x}{\left(c_{i} \cdot x+d_{i}\right)^{2}}\left(\frac{(-1)^{i+1}}{\beta-\frac{a_{i} \cdot x+b_{i}}{c_{i} \cdot x+d_{i}}}\right)^{\alpha},
$$

where $a_{i}, b_{i}, c_{i}, d_{i} \in \mathbb{Z}$, for $i \in\{1,2\}$, are such that

$$
f_{\nu}(x)=\frac{a_{1} \cdot x+b_{1}}{c_{1} \cdot x+d_{1}} \quad \text { and } \quad f_{\nu^{\prime}}(x)=\frac{a_{2} \cdot x+b_{2}}{c_{2} \cdot x+d_{2}} .
$$

The desired conclusion, namely that $\widehat{T}_{1}^{m}\left(v_{\beta, \alpha} \cdot \mathbb{1}_{[\nu] \cup\left[\nu^{\prime}\right]} / h_{1}\right)$ is of bounded variation follows from the following four observations.

1. For all $t \in(0,1]$, we have that $V_{[t, 1]}\left(\widehat{T}_{1}^{m}\left(v_{\beta, \alpha} \cdot \mathbb{1}_{[\nu] \cup\left[\nu^{\prime}\right]} / h_{1}\right)\right)<+\infty$.

2. For $i \in\{1,2\}$, by L'Hôpital's rule we have that

$$
\lim _{x \rightarrow 0} \frac{(-1)^{i+1} \cdot x}{\beta-\frac{a_{i} \cdot x+b_{i}}{c_{i} \cdot x+d_{i}}}=d_{i}^{2} .
$$

3. By L'Hôpital's rule, we have that

$$
\begin{aligned}
\lim _{x \rightarrow 0} \widehat{T}_{1}^{m}\left(v_{\beta, \alpha} \cdot \mathbb{1}_{[\nu] \cup\left[\nu^{\prime}\right]} / h_{1}\right)(x) \\
=\sum_{i=1}^{2} \lim _{x \rightarrow 0} \frac{x}{\left(c_{i} \cdot x+d_{i}\right)^{2}}\left(\frac{(-1)^{i+1}}{\beta-\frac{a_{i} \cdot x+b_{i}}{c_{i} \cdot x+d_{i}}}\right)^{\alpha}=0 .
\end{aligned}
$$

4. We have that

$$
\begin{aligned}
\frac{\mathrm{d}}{\mathrm{d} x} & \widehat{T}_{1}^{m}\left(v_{\beta, \alpha} \cdot \mathbb{1}_{[\nu] \cup\left[\nu^{\prime}\right]} / h_{1}\right)(x) \\
= & \sum_{i=1}^{2} \frac{\mathrm{d}}{\mathrm{d} x} \frac{x}{\left(c_{i} \cdot x+d_{i}\right)^{2}}\left(\frac{(-1)^{i+1}}{\beta-\frac{a_{i} \cdot x+b_{i}}{c_{i} \cdot x+d_{i}}}\right)^{\alpha} \\
= & \sum_{i=1}^{2} \frac{-c_{i} \cdot x+d_{i}}{\left(c_{i} \cdot x+d_{i}\right)^{3}}\left(\frac{(-1)^{i+1}}{\beta-\frac{a_{i} \cdot x+b_{i}}{c_{i} \cdot x+d_{i}}}\right)^{\alpha} \\
& -\frac{(-1)^{i+1} \cdot \alpha \cdot x}{\left(c_{i} \cdot x+d_{i}\right)^{4}}\left(\frac{(-1)^{i+1}}{\beta-\frac{a_{i} \cdot x+b_{i}}{c_{i} \cdot x+d_{i}}}\right)^{\alpha+1}
\end{aligned}
$$

which is non-negative on an open neighbourhood of zero.

The case when $\beta=1$ is a simplification of the above case.

Proof of Theorem 3.2 for $\beta$ irrational of intermediate $\alpha$-type. By linearity of the Perron-Frobenius operator we have that

$$
\ln (n) \cdot \mathcal{P}_{1}^{n}(v)=\ln (n) \cdot \mathcal{P}_{1}^{n}\left(v \cdot \mathbb{1}_{[0,1] \backslash\left[\left.\vartheta_{1}(\beta)\right|_{n}\right]}\right)+\ln (n) \cdot \mathcal{P}_{1}^{n}\left(v \cdot \mathbb{1}_{\left[\left.\vartheta_{1}(\beta)\right|_{n}\right]}\right) .
$$

Further, by Lemma 4.12 and the fact that $h_{1} \cdot \widehat{T}_{1}(f)=\mathcal{P}_{1}\left(f \cdot h_{1}\right)$, we have that

$$
\lim _{n \rightarrow \infty} \ln (n) \cdot \widehat{T}_{1}^{n}\left(v \cdot \mathbb{1}_{[0,1] \backslash\left[\left.\vartheta_{1}(\beta)\right|_{n}\right]} / h_{1}\right)=\int v \mathrm{~d} \lambda
$$


uniformly on compact subsets of $(0,1)$. Moreover, by the facts that $v \in \mathfrak{U}_{\beta, \alpha}$ is non-negative and $\mathcal{P}_{1}$ is a positive linear operator, there exists a positive constant $C$ with

$$
0 \leq \lim _{n \rightarrow \infty} \ln (n) \cdot \mathcal{P}_{1}^{n}\left(v \cdot \mathbb{1}_{\left[\left.\vartheta_{1}(\beta)\right|_{n}\right]}\right) \leq \lim _{n \rightarrow \infty} \ln (n) \cdot C \cdot v^{n, 1},
$$

where we recall that $v^{n, 1}:=\mathcal{P}_{1}^{n}\left(v \cdot \mathbb{1}_{\left[\left.\vartheta_{1}(\beta)\right|_{n}\right]}\right)$.

By Lemma 4.13, this latter limit is equal to zero outside a set of Hausdorff dimension equal to zero.

All that remains to show is that if $\beta \in(0,1]$ is irrational, pre-periodic with respect to $T_{1}$ and has period length strictly greater than one, then on $\omega_{1}(\beta)$ we have that

$$
\liminf _{n \rightarrow+\infty} \ln (n) \cdot \mathcal{P}_{1}^{n}(v)=\int v \mathrm{~d} \lambda \cdot h_{1} \quad \text { and } \quad \limsup _{n \rightarrow+\infty} \ln (n) \cdot \mathcal{P}_{1}^{n}(v)=+\infty
$$

and in the case that $\beta \in(0,1]$ is pre-periodic with respect to $T_{1}$ and has period length equal to one then on the singleton $\omega_{1}(\beta)$ we have that the limit in (4) is equal to $+\infty$.

By positivity and linearity of $\mathcal{P}_{1}^{n}$ and Lemma 4.12, it suffices to show, if $\beta \in(0,1]$ is irrational, pre-periodic with respect to $T_{1}$ and has period length strictly greater than one, then on $\omega_{1}(\beta)$,

$$
\liminf _{n \rightarrow+\infty} \ln (n) \cdot v^{n, 1}=0 \quad \text { and } \quad \limsup _{n \rightarrow+\infty} \ln (n) \cdot v^{n, 1}=+\infty
$$

and in the case that $\beta \in(0,1]$ is pre-periodic with respect to $T_{1}$ and has period length equal to one, then on the singleton $\omega_{1}(\beta)$,

$$
\lim _{n \rightarrow+\infty} \ln (n) \cdot v^{n, 1}=+\infty .
$$

Indeed if $\beta$ is pre-periodic with respect to $T_{1}$ and has period length $l \geq 1$, then letting $n \in \mathbb{N}_{0}$, be the minimal integer so that $T_{1}^{n+k}(\beta)=T_{1}^{n+k+l}(\bar{\beta})$, for all $k \in \mathbb{N}_{0}$, we have that

$$
f_{1,\left(\vartheta_{1, n+j+1}(\beta), \ldots, \vartheta_{1, n+j+l}(\beta)\right)}\left(T_{1}^{n+j}(\beta)\right)=T_{1}^{n+j}(\beta),
$$

for all $j \in\{0,1, \ldots, l-1\}$. Further, $\omega_{1}(\beta)=\left\{T_{1}^{n}(\beta), \ldots, T_{1}^{n+l-1}(\beta)\right\}$, and hence, for $j \in\{0,1, \ldots, l-1\}$, it follows that $v^{n+j+k \cdot l, 1}\left(T_{1}^{n+j}(\beta)\right)=+\infty$, for all $k \in \mathbb{N}_{0}$. To complete the proof we will show, for $l>1$ and $i, j \in\{0,1, \ldots, l-1\}$ with $i \neq j$, that

$$
\lim _{k \rightarrow+\infty} v^{n+j+k \cdot l, 1}\left(T_{1}^{n+i}(\beta)\right)=0 .
$$

To this end set $L:=\min \left\{\mid T_{1}^{n+j}(\beta)-T_{1}^{n+i}(\beta): i, j \in\{0,1, \ldots, l-1\}\right.$ and $\left.i \neq j\right\}$ and set

$$
a:=\min \left\{T_{1}^{n+j}(\beta): j \in\{0,1, \ldots, l-1\}\right\}
$$

and

$$
b:=\max \left\{T_{1}^{n+j}(\beta): j \in\{0,1, \ldots, l-1\}\right\} .
$$


Since $\beta$ is irrational and pre-periodic with period $m>1$, it follows that $0<$ $a<b<1$ and therefore,

$$
\begin{aligned}
& \left|f_{1,\left.\vartheta_{1}(\beta)\right|_{n+j+k \cdot l}}^{\prime}\left(T_{1}^{n+i}(\beta)\right)\right| \\
& \leq a^{-2}\left((r(n+j+k \cdot l)+1) q_{m(n+j+k \cdot l)}+q_{m(n+j+k \cdot l)-1}\right)^{-2}
\end{aligned}
$$

for all $i, j \in\{0,1, \ldots, l-1\}$ and $k \in \mathbb{N}$. Further, we have that

$$
\begin{aligned}
\mid \beta & -f_{1,\left.\vartheta_{1}(\beta)\right|_{n+j+k \cdot l}}\left(T_{1}^{n+i}(\beta)\right) \mid \\
& \geq\left|f_{1,\left.\vartheta_{1}(\beta)\right|_{n+j+k \cdot l}}\left(T_{1}^{n+j+k \cdot l}(\beta)\right)-f_{1,\left.\vartheta_{1}(\beta)\right|_{n+j+k \cdot l}}\left(T_{1}^{n+i}(\beta)\right)\right| \\
& \geq \inf _{u \in[a, b]}\left|f_{1,\left.\vartheta_{1}(\beta)\right|_{n+j+k \cdot l}}^{\prime}(u)\right| \cdot\left|T_{1}^{n+j}(\beta)-T_{1}^{n+i}(\beta)\right| \\
& \geq\left((r(n+j+k \cdot l)+1) q_{m(n+j+k \cdot l)}+q_{m(n+j+k \cdot l)-1}\right)^{-2} \cdot L,
\end{aligned}
$$

for all $i, j \in\{0,1, \ldots, l-1\}$ with $i \neq j$ and $k \in \mathbb{N}$. Hence, for all $i, j \in$ $\{0,1, \ldots, l-1\}$ with $i \neq j$, we have

$$
\begin{aligned}
0 \leq & \lim _{l \rightarrow+\infty} v^{n+j+l \cdot m, 1}\left(T_{1}^{n+i}(\beta)\right) \\
\leq & \lim _{l \rightarrow+\infty}\left|f_{1,\left.\vartheta_{1}(\beta)\right|_{n+j+l \cdot m}}^{\prime}\left(T_{1}^{n+i}(\beta)\right)\right| \cdot\left|\beta-f_{1,\left.\vartheta_{1}(\beta)\right|_{n+j+l \cdot m}}\left(T_{1}^{n+i}(\beta)\right)\right|^{-\alpha} \\
\leq & \lim _{l \rightarrow+\infty} a^{-2} \cdot L^{-\alpha} \cdot\left((r(n+j+k \cdot l)+1) q_{m(n+j+k \cdot l)}\right. \\
& \left.+q_{m(n+j+k \cdot l)-1}\right)^{2 \cdot(\alpha-1)}=0 .
\end{aligned}
$$

This completes the proof.

\subsubsection{Proof of Theorem 3.3.}

Proof of Theorem 3.3(a). Within this proof set

$$
\beta=[0 ; \underbrace{1,1,2}_{2 \cdot 1}, \underbrace{1,1,1,1}_{2 \cdot 2}, 2, \underbrace{1,1,1,1,1,1}_{2 \cdot 3}, 2, \ldots]
$$

and

$$
\kappa=[0 ; \underbrace{1,1,1}_{2^{1}}, 2 \underbrace{1,1,1,1}_{2^{2}}, 2, \underbrace{1,1, \ldots, 1}_{2^{3}}, 2, \ldots]
$$

and, for $n \in \mathbb{N}$, set

$$
\Lambda(n, \tau):= \begin{cases}n \cdot(n+2) & \text { if } \tau=\beta, \\ 2^{n}+n-2 & \text { if } \tau=\kappa .\end{cases}
$$

Observe that $\beta, \kappa \in[1 / 2,1]$. Letting $a_{n}(\beta)$ and $a_{n}(\kappa)$ denote the $n$th continued fraction entry of $\beta$ and $\kappa$, respectively, an elementary calculation yields that $a_{\Lambda(n, \beta)-1}(\beta)=a_{\Lambda(n, \kappa)-1}(\kappa)=2$. Further, one can show that

$$
\omega_{1}(\beta)=\omega_{1}(\kappa)=\left\{[0 ; \underbrace{1,1, \ldots, 1}_{k}, 2, \overline{1}]: k \in \mathbb{N}_{0}\right\} \cup\{\gamma:=(\sqrt{5}-1) / 2=[0 ; \overline{1}]\} .
$$


Recall from (9) that $v_{\tau, \alpha, n, 1}=\left|f_{1,\left.\vartheta_{1}(\tau)\right|_{n}}^{\prime}\right| \cdot\left|\tau-f_{1,\left.\vartheta_{1}(\tau)\right|_{n}}\right|^{-\alpha}$. Following the same arguments as in beginning of the proof of Theorem 3.2, it is sufficient to show, on $\omega_{1}(\beta)=\omega_{1}(\kappa)$, that

$$
\limsup _{n \rightarrow+\infty} \ln (n) \cdot v_{\beta, \alpha, n, 1}=0
$$

and

$$
\limsup _{n \rightarrow+\infty} \ln (n) \cdot v_{\kappa, \alpha, n, 1}= \begin{cases}0 & \text { if } \alpha \in(0,1 / 2), \\ +\infty & \text { if } \alpha \in(1 / 2,1] .\end{cases}
$$

To this end fix $k \in \mathbb{N}_{0}$ and set

$$
\zeta_{k}:=[0 ; \underbrace{1,1, \ldots, 1}_{k}, 2, \overline{1}] \in[1 / 3,1] .
$$

We will show that the equalities given in (20) hold for $\zeta_{k}$, the result for $\gamma$ is a simplification of this case. To this end let $\tau \in\{\beta, \kappa\}$. By the mean value theorem, for each $n \in \mathbb{N}$, there exists $u_{n}(\tau) \in(1 / 3,1)$ such that

$$
\begin{aligned}
\mid \tau & -f_{1,\left.\vartheta_{1}(\tau)\right|_{n}\left(\zeta_{k}\right) \mid} \\
& =\left|T_{1}^{n}(\tau)-\zeta_{k}\right| \cdot\left|f_{1,\left.\vartheta_{1}(\tau)\right|_{n}}^{\prime}\left(u_{n}(\tau)\right)\right| \\
& =\left|T_{1}^{n}(\tau)-\zeta_{k}\right| \cdot\left(\left(r(n, \tau) u_{n}(\tau)+1\right) q_{m(n, \tau)}(\tau)+q_{m(n, \tau)-1}(\tau) u_{n}(\tau)\right)^{-2} \\
& \left\{\begin{array}{l}
\geq 5^{-2} \cdot\left(q_{m(n, \tau)}(\tau)\right)^{-2} \cdot\left|T_{1}^{n}(\tau)-\zeta_{k}\right|, \\
\leq\left(q_{m(n, \tau)}(\tau)\right)^{-2} \cdot\left|T_{1}^{n}(\tau)-\zeta_{k}\right|,
\end{array}\right.
\end{aligned}
$$

where $m(n, \tau)$ and $r(n, \tau)$ are as defined in (14) and where, for $l \in \mathbb{N}_{0}$, the integers $p_{l}(\tau)$ and $q_{l}(\tau)$ are as defined in (3). Thus, for $\tau \in\{\beta, \kappa\}$ and $k \in \mathbb{N}_{0}$, we have that

$$
\begin{aligned}
& \limsup _{n \rightarrow \infty} \ln (n) \cdot v_{\tau, \alpha, n, 1}\left(\zeta_{k}\right) \\
& =\limsup _{n \rightarrow \infty} \frac{\ln (n)}{\left(\left(r(n, \tau) \cdot \zeta_{k}+1\right) \cdot q_{m(n, \tau)}(\tau)+q_{m(n, \tau)-1} \cdot \zeta_{k}\right)^{2}} \frac{1}{\left|\tau-f_{1,\left.\vartheta_{1}(\tau)\right|_{n}}\left(\zeta_{k}\right)\right|^{\alpha}} \\
& \left\{\begin{array}{l}
\geq \limsup _{n \rightarrow \infty} \frac{\ln (n)}{5^{2} \cdot\left(q_{m(n, \tau)}(\tau)\right)^{2 \cdot(1-\alpha)}} \frac{1}{\left|T_{1}^{n}(\tau)-\zeta_{k}\right|^{\alpha}} \\
\leq \limsup _{n \rightarrow \infty} \frac{5^{2 \cdot \alpha} \cdot \ln (n)}{\left(q_{m(n, \tau)}(\tau)\right)^{2 \cdot(1-\alpha)}} \frac{1}{\left|T_{1}^{n}(\tau)-\zeta_{k}\right|^{\alpha}}
\end{array}\right. \\
& \left\{\begin{array}{l}
\geq \limsup _{n \rightarrow \infty} \frac{\ln (n)}{5^{2} \cdot\left(q_{m(n, \tau)}(\tau)\right)^{2 \cdot(1-\alpha)}} \frac{1}{\left|T_{1}^{n-(k+1)}(\tau)-\gamma\right|^{\alpha}} \frac{1}{\left|\left(f_{1,1}^{k} \circ f_{1,0} \circ f_{1,1}\right)^{\prime}(0)\right|^{\alpha}} \\
\leq \limsup _{n \rightarrow \infty} \frac{5^{2 \cdot \alpha} \cdot \ln (n)}{\left(q_{m(n, \tau)}(\tau)\right)^{2 \cdot(1-\alpha)}} \frac{1}{\left|T_{1}^{n-(k+1)}(\tau)-\gamma\right|^{\alpha}} \frac{1}{\left|\left(f_{1,1}^{k} \circ f_{1,0} \circ f_{1,1}\right)^{\prime}(1)\right|^{\alpha}} .
\end{array}\right.
\end{aligned}
$$

Hence it is sufficient to show that, for $\alpha \in(0,1)$,

$$
\limsup _{n \rightarrow+\infty} \frac{\ln (n)}{\left(q_{m(n, \beta)}(\beta)\right)^{2 \cdot(1-\alpha)}} \frac{1}{\left|T_{1}^{n-(k+1)}(\beta)-\gamma\right|^{\alpha}}=0
$$


and

$$
\limsup _{n \rightarrow+\infty} \frac{\ln (n)}{\left(q_{m(n, \kappa)}(\kappa)\right)^{2 \cdot(1-\alpha)}} \frac{1}{\left|T_{1}^{n-(k+1)}(\kappa)-\gamma\right|^{\alpha}}= \begin{cases}0 & \text { if } \alpha \in(0,1 / 2), \\ +\infty & \text { if } \alpha \in(1 / 2,1)\end{cases}
$$

We will first show the equality given in (21) after which we will show the equality given in (22). For this observe that if $n-(k+1)=\Lambda(l, \beta)+(l-1)$, for some $l \in \mathbb{N}$, then

$$
T_{1}^{n-(k+1)}(\beta)=[0 ; 2, \underbrace{1,1, \ldots, 1}_{2 \cdot(l+1)}, 2, \underbrace{1,1, \ldots, 1}_{2 \cdot(l+2)}, 2, \underbrace{1,1, \ldots, 1}_{2 \cdot(l+3)}, \ldots] \in[1 / 3,1 / 2],
$$

and hence,

$$
\begin{aligned}
& \frac{\ln (n)}{\left(q_{m(n, \beta)}(\beta)\right)^{2 \cdot(1-\alpha)}} \frac{1}{\left|T_{1}^{n-(k+1)}(\beta)-\gamma\right|^{\alpha}} \\
& \quad \leq \frac{\ln (\Lambda(l, \beta)+(l-1)+(k+1))}{\left(q_{\Lambda(l, \beta)}(\beta)\right)^{2 \cdot(1-\alpha)}} \frac{1}{|(1 / 2)-\gamma|^{\alpha}} \\
& \quad \sim \frac{2 \cdot \ln (l)}{\left(q_{l \cdot(l+2)}(\beta)\right)^{2 \cdot(1-\alpha)}} \frac{1}{|(1 / 2)-\gamma|^{\alpha}} .
\end{aligned}
$$

Since the sequence $\left(q_{j}\right)_{j \in \mathbb{N}}$ grows exponentially, this latter term converges to zero as $l \rightarrow \infty$. (Here we have used the fact that $n-(k+1)=\Lambda(l, \beta)+(l-1)$.)

In the case that $n-(k+1) \notin\{\Lambda(j, \beta)+(j-1): j \in \mathbb{N}\}$, set $l=l(n) \in \mathbb{N}$ to be the maximal integer such that $n-(k+1)>\Lambda(l, \beta)+(l-1)$, in which case

$$
T_{1}^{n-(k+1)}(\beta)=[0 ; \underbrace{1,1, \ldots \ldots \ldots \ldots \ldots, 1}_{\begin{array}{c}
3 \cdot(l+1)+(k+1)+\Lambda(l, \beta)-n \\
\leq 2 \cdot(l+1)+1
\end{array}}, 2 \underbrace{1,1, \ldots, 1}_{2 \cdot(l+2)}, 2, \underbrace{1,1, \ldots, 1}_{2 \cdot(l+3)}, \ldots],
$$

and hence,

$$
\begin{aligned}
\left.\frac{\ln (n)}{\left(q_{m}(n, \beta)\right.}(\beta)\right)^{2 \cdot(1-\alpha)} & \frac{1}{\left|T_{1}^{n-(k+1)}(\beta)-\gamma\right|^{\alpha}} \\
= & \frac{\ln (n)}{\left(q_{m(n, \beta)}(\beta)\right)^{2 \cdot(1-\alpha)}} \\
& \frac{\left|f_{1,1}^{3 \cdot(l+1)+(k+1)+\Lambda(l, \beta)-n}\left(T_{1}^{\Lambda(l+1, \beta)+l}(\beta)\right)-f_{1,1}^{3 \cdot(l+1)+(k+1)+\Lambda(l, \beta)-n}(\gamma)\right|^{\alpha}}{1} \\
\leq & \frac{\ln ((l+2) \cdot(l+5))}{\left(q_{l \cdot(l+2)}(\beta)\right)^{2 \cdot(1-\alpha)}} \frac{1}{\inf _{u \in[0,1]}\left|\left(f_{1,1}^{3 \cdot(l+1)+(k+1)+\Lambda(l, \beta)-n}\right)^{\prime}(u)\right|^{\alpha}} \frac{1(1 / 2)-\left.\gamma\right|^{\alpha}}{|(1 / 2)-\gamma|^{\alpha}} \\
= & \frac{\ln ((l+2) \cdot(l+5))}{\left(q_{l \cdot(l+2)}(\beta)\right)^{2 \cdot(1-\alpha)}} \frac{\left(q_{3 \cdot(l+1)+(k+1)+\Lambda(l, \beta)-n}(\gamma)\right)^{\alpha}}{\mid} \\
= & \frac{\ln ((l+2) \cdot(l+5))}{\left(q_{l \cdot(l+2)}(\beta)\right)^{2 \cdot(1-\alpha)}} \frac{\left(q_{2 \cdot(l+1)+1}(\beta)\right)^{\alpha}}{|(1 / 2)-\gamma|^{\alpha}} .
\end{aligned}
$$


Since the sequence $\left(q_{j}(\beta)\right)_{j \in \mathbb{N}}$ grows exponentially, this latter term converges to zero as $l=l(n) \rightarrow \infty$. The equality stated in (21) now follows from (23) and (24).

We will now prove the equality given in (22). The result for, $\alpha \in(0,1 / 2)$, follows in a similar manner to the previous case. Indeed, observe that if $n-$ $(k+1)=\Lambda(l, \kappa)+(l-1)$, for some $l \in \mathbb{N}$, then

$$
T_{1}^{n-(k+1)}(\kappa)=[0 ; 2, \underbrace{1,1, \ldots, 1}_{2^{l+1}}, 2, \underbrace{1,1, \ldots, 1}_{2^{l+2}}, 2, \underbrace{1,1, \ldots, 1}_{2^{l+3}}, 2, \ldots] \in[1 / 3,1 / 2],
$$

and hence, for $n$ sufficiently large,

$$
\frac{\ln (n)}{\left(q_{m(n, \kappa)}(\kappa)\right)^{2 \cdot(1-\alpha)}} \frac{1}{\left|T_{1}^{n-(k+1)}(\kappa)-\gamma\right|^{\alpha}} \leq \frac{(l+1) \cdot \ln (2)}{\left(q_{2^{l}}(\kappa)\right)^{2 \cdot(1-\alpha)}} \frac{1}{|(1 / 2)-\gamma|^{\alpha}} .
$$

The sequence $\left(q_{j}(\kappa)\right)_{j \in \mathbb{N}}$ grows exponentially, in particular there exists a positive constant $c$ so that $\kappa^{-j} / c \leq q_{j}(\kappa) \leq c \cdot \kappa^{-j}$. Therefore, the latter term in (25) converges to zero as $l \rightarrow \infty$. (Here we have used the fact that $n-(k+1)=$ $\Lambda(l, \kappa)+(l-1)$.

In the case that $n-(k+1) \notin\{\Lambda(j, \kappa)+(j-1): j \in \mathbb{N}\}$, set $l=l(n) \in \mathbb{N}$ to be the maximal integer such that $n-(k+1)>\Lambda(l, \kappa)+(l-1)$, in which case

$$
T_{1}^{n-(k+1)}(\kappa)=[0 ; \underbrace{1,1, \ldots \ldots \ldots \ldots \ldots, 1}_{2^{l+1}+(l+1)+(k+1)+\Lambda(l, \kappa)-n}, 2, \underbrace{1,1, \ldots, 1}_{2^{l+1}+1}, 2, \underbrace{1,1, \ldots, 1}_{2^{l+3}}, 2, \ldots] .
$$

We also observe that $q_{i}(\gamma) \leq q_{i}(\kappa)$, for all $i \in \mathbb{N}_{0}$. Therefore, it follows that

$$
\begin{aligned}
\frac{\ln (n)}{\left(q_{m(n, \kappa)}(\kappa)\right)^{2 \cdot(1-\alpha)} \frac{1}{\left|T_{1}^{n-(k+1)}(\kappa)-\gamma\right|^{\alpha}}} & \leq \frac{(l+2) \cdot \ln (2)}{\left(q_{2^{l}}(\kappa)\right)^{2 \cdot(1-\alpha)}}\left(q_{2 \cdot 2^{l}+2}(\gamma)\right)^{\alpha} \\
& \leq \frac{(l+2) \cdot \ln (2)}{\left(q_{2^{l}}(\gamma)\right)^{2 \cdot(1-\alpha)} \cdot\left(q_{2 \cdot 2^{l}+2}(\gamma)\right)^{-\alpha}}
\end{aligned}
$$

Since there exists a positive constant $c$ so that $\gamma^{-j} / c \leq q_{j}(\gamma) \leq c \cdot \gamma^{-j}$, if $\alpha \in(0,1 / 2)$, this latter term converges to zero as $l=l(n) \rightarrow \infty$. The equality in $(22)$ for $\alpha \in(0,1 / 2)$ follows from $(25)$ and $(26)$.

Let us now examine the case that $\alpha \in(1 / 2,1)$. It follows from an inductive argument that, for all $n \in \mathbb{N}, q_{l}(\kappa) \leq 2^{n} \cdot q_{l}(\gamma)$ for all integers $l \in[\Lambda(n, \kappa), \Lambda(n+$ $1, \kappa)$ ). Further, for all $n \in \mathbb{N}$ we have that

1. $\left|\gamma-T_{1}^{\Lambda(n, \kappa)+n-1}(\kappa)\right|=\left|\gamma-[0 ; 2, \underbrace{1, \ldots, 1}_{2^{n+1}}, 2, \underbrace{1, \ldots, 1}_{2^{n+2}}, \ldots]\right| \geq|\gamma-(1 / 2)|$

and

2. $\left|\gamma-T_{1}^{\Lambda(n, \kappa)+n+1}(\kappa)\right|=\left|\gamma-[0 ; \underbrace{1, \ldots, 1}_{2^{n}}, 2, \underbrace{1, \ldots, 1}_{2^{n+1}}, 2, \ldots]\right|$

$\leq\left|\gamma-\frac{p_{2^{n}}(\gamma)}{q_{2^{n}}(\gamma)}\right| \leq \frac{1}{\left(q_{2^{n}}(\gamma)\right)^{2}}$ 
Therefore, if $\alpha \in(1 / 2,1)$, since there exists a positive constant $\mathfrak{s}$ so that $\gamma^{-n} / \mathfrak{s} \leq q_{n}(\gamma) \leq \mathfrak{s} \cdot \gamma^{-n}$, for all $n \in \mathbb{N}$, we have that

$$
\begin{aligned}
& \limsup _{n \rightarrow+\infty} \frac{\ln (\Lambda(n, \kappa)+n+1)}{\left(q_{\Lambda(n, \kappa)}(\kappa)\right)^{2 \cdot(1-\alpha)}} \frac{1}{\left|T_{1}^{\Lambda(n, \kappa)+n+1}(\kappa)-\gamma\right|^{\alpha}} \\
& \quad \geq \limsup _{n \rightarrow+\infty} \frac{n \cdot \ln (2) \cdot\left(q_{2^{n}}(\gamma)\right)^{2 \cdot \alpha}}{2^{2 \cdot n \cdot(1-\alpha)} \cdot\left(q_{2^{n}+n-2}(\gamma)\right)^{2 \cdot(1-\alpha)}} \\
& \quad \geq \limsup _{n \rightarrow+\infty} \frac{n \cdot \ln (2)}{\mathfrak{s}^{2} \cdot \gamma^{2^{n+1} \cdot(2 \cdot \alpha-1)+2 \cdot(4 \cdot n-4) \cdot(1-\alpha)}}=+\infty .
\end{aligned}
$$

Moreover, since the sequence $\left(q_{j}(\kappa)\right)_{j \in \mathbb{N}}$ grows exponentially, it follows that

$$
\begin{gathered}
\liminf _{n \rightarrow+\infty} \frac{\ln (\Lambda(n, \kappa)+n-1)}{\left(q_{\Lambda(n, \kappa)-1}(\kappa)\right)^{2 \cdot(1-\alpha)}} \frac{1}{\left|T_{1}^{\Lambda(n, \kappa)+n-1}(\kappa)-\gamma\right|^{\alpha}} \\
\leq \liminf _{n \rightarrow+\infty} \frac{\ln (\Lambda(n, \kappa)+n-1)}{\left(q_{\Lambda(n, \kappa)-1}(\kappa)\right)^{2 \cdot(1-\alpha)}} \frac{1}{|\gamma-(1 / 2)|^{\alpha}}=0 .
\end{gathered}
$$

This completes the proof.

Proof of Theorem 3.3(b). Since $\lim _{n \rightarrow+\infty} a_{n}=+\infty$, we have that $\omega_{1}(\beta)=$ $\{1 / k: k \in \mathbb{N}\} \cup\{0\}$. Let $v_{\beta, \alpha, n, 1}$ be as in (9). Following the same arguments as in beginning of the proof of Theorem 3.2, it is sufficient to show, for a fixed $k \in \mathbb{N}$, that

$$
\limsup _{n \rightarrow+\infty} \ln (n) \cdot v_{\beta, \alpha, n, 1}(1 / k) \begin{cases}=0 & \text { if } \limsup _{j \rightarrow \infty} \mathscr{S}_{k, j}=0, \\ >0 & \text { if } \limsup _{j \rightarrow \infty} \mathscr{S}_{k, j}>0 .\end{cases}
$$

and

$$
\liminf _{n \rightarrow+\infty} \ln (n) \cdot v_{\beta, \alpha, n, 1}(1 / k)=0
$$

To this end fix $k \in \mathbb{N}$ and, for $n \in \mathbb{N}$, set $z=z(n):=T_{1}^{n}(\beta)$. (Note, $z$ is the unique real number in $[0,1]$ such that $f_{1,\left.\vartheta_{1}(\beta)\right|_{n}}(z)=\beta$.) If $z \in(1 /(k+1), 1 / k)$, then, by the mean value theorem, there exists $u=u(n) \in(1 /(k+1), 1 / k)$ such that

$$
\begin{aligned}
\mid \beta & -f_{1,\left.\vartheta_{1}(\beta)\right|_{n}(1 / k) \mid} \\
& =\left|f_{1,\left.\vartheta_{1}(\beta)\right|_{n}}(z)-f_{1,\left.\vartheta_{1}(\beta)\right|_{n}}(1 / k)\right| \\
& =|1 / k-z| \cdot\left|f_{1,\left.\vartheta_{1}(\beta)\right|_{n}}^{\prime}(u)\right| \\
& =\left|1 / k-T_{1}^{n}(\beta)\right| \cdot\left|(r(n) \cdot u+1) \cdot q_{m(n)}+q_{m(n)-1} \cdot u\right|^{-2} \\
& \left\{\begin{array}{l}
\geq k^{2} \cdot\left|1 / k-T_{1}^{n}(\beta)\right| \cdot\left|(r(n)+k) \cdot q_{m(n)}+q_{m(n)-1}\right|^{-2}, \\
\leq(k+1)^{2} \cdot\left|1 / k-T_{1}^{n}(\beta)\right| \cdot\left|(r(n)+k+1) \cdot q_{m(n)}+q_{m(n)-1}\right|^{-2} .
\end{array}\right.
\end{aligned}
$$


If $z \notin(1 /(k+1), 1 / k)$, then, since $f_{1,\left.\vartheta_{1}(\beta)\right|_{n}}$ is either order preserving or order reversing, we have for $n \in \mathbb{N}$ sufficiently large that

$$
\begin{aligned}
\mid \beta & -f_{1,\left.\vartheta_{1}(\beta)\right|_{n}(1 / k) \mid} \\
& =\mid f_{1,\left.\vartheta_{1}(\beta)\right|_{n}(z)-f_{1,\left.\vartheta_{1}(\beta)\right|_{n}}(1 / k) \mid} \\
& \geq \begin{cases}\left|f_{1,\left.\vartheta_{1}(\beta)\right|_{n}}(1 / 2)-f_{1,\left.\vartheta_{1}(\beta)\right|_{n}}(1)\right| & \text { if } k=1, \\
\min \left\{\left|f_{1,\left.\vartheta_{1}(\beta)\right|_{n}}(1 /(k+1))-f_{1,\left.\vartheta_{1}(\beta)\right|_{n}}(1 / k)\right|,\right. & \\
\left.\left|f_{1,\left.\vartheta_{1}(\beta)\right|_{n}}((2 k-1) /(2 k(k-1)))-f_{1,\left.\vartheta_{1}(\beta)\right|_{n}}(1 / k)\right|\right\} & \text { otherwise. }\end{cases}
\end{aligned}
$$

By the mean value theorem there exists $u \in(1 /(k+1),(2 k-1) /(2 k(k-1)))$ if $k \neq 1$ and $u \in(1 / 2,1)$ if $k=1$ such that

$$
\begin{aligned}
\left|\beta-f_{1,\left.\vartheta_{1}(\beta)\right|_{n}}(x)\right| & \geq(2 \cdot k \cdot(k+1))^{-1} \cdot\left|f_{1,\left.\vartheta_{1}(\beta)\right|_{n}}^{\prime}(u)\right| \\
& \geq(6 \cdot k)^{-1} \cdot\left|(r(n)+\max \{k-1,1\}) \cdot q_{m(n)}+q_{m(n)-1}\right|^{-2} .
\end{aligned}
$$

We now consider the following cases $z \notin(1 /(k+1), 1 / k)$ and $z \in(1 /(k+$ $1), 1 / k)$.

1. If $z \notin(1 /(k+1), 1 / k)$, then

$$
\begin{aligned}
0 & \leq \ln (n) \cdot v_{\beta, \alpha, n, 1}(1 / k) \\
& =\frac{\ln (n)}{\left((r(n) / k+1) \cdot q_{m(n)}+q_{m(n)-1} / k\right)^{2}} \frac{1}{\left|\beta-f_{1,\left.\vartheta_{1}(\beta)\right|_{n}}(1 / k)\right|^{\alpha}} \\
& \leq \frac{6^{2 \cdot \alpha} \cdot k^{2 \cdot(1-\alpha)} \cdot \ln (n)}{\left((r(n)+1) \cdot q_{m(n)}+q_{m(n)-1}\right)^{2 \cdot(1-\alpha)}} .
\end{aligned}
$$

As $(r(n)+1) \cdot q_{m(n)}+q_{m(n)-1}>n$, for all $n \in \mathbb{N}$, it follows that $\liminf _{n \rightarrow+\infty} \ln (n) \cdot v_{\beta, \alpha, n, 1}(1 / k)=0$.

2. If $z \in(1 /(k+1), 1 / k)$, then $z=T_{1}^{n}(\beta)=\left[0 ; k, a_{m(n)}, a_{m(n)+1}, \ldots\right]$; that is $n=n_{k, m(n)}$. Thus, we have that

$$
\begin{aligned}
& \limsup _{j \rightarrow+\infty} \ln \left(n_{k, j}\right) \cdot v_{\beta, \alpha, n_{k, j}, 1}(1 / k) \\
& \quad=\limsup _{j \rightarrow+\infty} \frac{k^{2} \cdot \ln \left(n_{k, j}\right)}{\left(\left(r\left(n_{k, j}\right)+k\right) \cdot q_{m\left(n_{k, j}\right)}+q_{m\left(n_{k, j}\right)-1}\right)^{2}} \frac{1}{\left|\beta-f_{1,\left.\vartheta_{1}(\beta)\right|_{n_{k, j}}}(1 / k)\right|^{\alpha}} \\
& \left\{\begin{array}{l}
\leq \limsup _{j \rightarrow+\infty} \frac{k^{2 \cdot(1-\alpha)} \cdot \ln \left(n_{k, j}\right)}{\left|1 / k-T_{1}^{n_{k, j}}(\beta)\right|^{\alpha} \cdot\left(\left(r\left(n_{k, j}\right)+k\right) \cdot q_{m\left(n_{k, j}\right)}+q_{m\left(n_{k, j}\right)-1}\right)^{2 \cdot(1-\alpha)}} \\
\geq \limsup _{j \rightarrow+\infty} \frac{k^{2 \cdot(1+\alpha)} \cdot \ln \left(n_{k, j}\right)}{2^{2 \cdot \alpha} \cdot\left|1 / k-T_{1}^{n_{k, j}}(\beta)\right|^{\alpha} \cdot\left(\left(r\left(n_{k, j}\right)+k\right) \cdot q_{m\left(n_{k, j}\right)}+q_{m\left(n_{k, j}\right)-1}\right)^{2 \cdot(1-\alpha)}}
\end{array}\right. \\
& \left\{\begin{array}{l}
\leq \limsup _{j \rightarrow+\infty} \frac{k^{2} \cdot\left(a_{j+1}+1\right)^{\alpha} \cdot \ln \left(n_{k, j}\right)}{\left(q_{j}\right)^{2 \cdot(1-\alpha)}=\limsup _{j \rightarrow+\infty} k^{2} \cdot \mathscr{S}_{k, j}} \\
\geq \limsup _{j \rightarrow+\infty} \frac{k^{2(1+2 \cdot \alpha)} \cdot\left(a_{j+1}\right)^{\alpha} \cdot \ln \left(n_{k, j}\right)}{2^{2 \cdot \alpha} \cdot\left(q_{j}\right)^{2 \cdot(1-\alpha)}}=\limsup _{j \rightarrow+\infty} k^{2 \cdot(1+2 \cdot \alpha)} \cdot 4^{-\alpha} \cdot \mathscr{S}_{k, j} \cdot
\end{array}\right.
\end{aligned}
$$

This completes the proof. 


\section{References}

[1] Aaronson, J.: An Introduction to Infinite Ergodic Theory. AMS Mathematical Surveys and Monographs, vol. 50 (1997)

[2] Aaronson, J.: An ergodic theorem with large normalising constants. Isr. J. Math. 38, 182-188 (1981)

[3] Baladi, V.: Positive Transfer Operators and Decay of Correlations. World Scientific, Singapore (2000)

[4] Boyarsky, A., Góra, P.: Laws of Chaos: Invariant Measures and Dynamical Systems in One Dimension. Probability and Its Applications. Birkhäuser, Basel (1997)

[5] Collet, P.: Some Ergodic Properties of Maps of the Interval, vol. 52. Dynamical systems (Temuco, 1991/1992), pp. 55-91, Travaux en Cours. Herman (1996)

[6] Dajani, K., Kraaikamp, C.: Ergodic Theory of Numbers. The Carus Mathematical Monographs, vol. 29 (2002)

[7] Dedecker, J., Gouëzel, S., Merlevède, F.: Some almost sure results for unbounded functions of intermittent maps and their associated Markov chains. Ann. Inst. H. Poincaré Probab. Stat. 46(3), 796-821 (2010)

[8] Doeblin, W., Fortet, R.: Sur des chaînes à liaisons complètes. Bull. Soc. Math. Fr. 65, 132-148 (1937)

[9] Erickson, K.: Strong renewal theorems with infinite mean. Trans. Am. Math. Soc. 151, 263-291 (1970)

[10] Esposti, M.D., Isola, S., Knauf, A.: Generalized Farey trees, transfer operators and phase transitions. Commun. Math. Phys. 275, 297-329 (2007)

[11] Falconer, K.J.: Fractal Geometry: Mathematical Foundations and Applications, 3th edn. Wiley, New York (2014)

[12] Fiala, J., Kleban, P., Özlük, A.: The phase transition in statistical models defined on Farey fractions. J. Stat. Phys. 110, 73-86 (2003)

[13] Fremlin, D.H.: Measure Theory, vol. 2. Torres Fremlin, Colchester (2001)

[14] Garsia, A., Lamperti, J.: A discrete renewal theorem with infinite mean. Comment. Math. Helv. 37, 221-234 (1962)

[15] Giampieri, M., Isola, S.: A one-parameter family of analytic Markov maps with an intermittency transition. Discrete Contin. Dyn. Syst. 12(1), 115-136 (2005)

[16] Gouëzel, S.: Sharp polynomial estimates for the decay of correlations. Isr. J. Math. 139, 29-65 (2004)

[17] Gouëzel, S.: Berry-Esseen theorem and local limit theorem for non uniformly expanding maps. Ann. Inst. H. Poincaré Probab. Stat. 41(6), 997-1024 (2005)

[18] Gouëzel, S.: Stable Laws for the Doubling Map. Unpublished, pp. 1-15 (2008)

[19] Gouëzel, S.: Correlation asymptotics from large deviations in dynamical systems with infinite measure. Colloq. Math. 125(2), 193-212 (2011)

[20] Hofbauer, F., Keller, G.: Ergodic properties of invariant measures for piecewise monotonic transformations. Math. Z. 180, 119-140 (1982)

[21] Hennion, H., Herveé, L.: Limit Theorems for Markov Chains and Stochastic Properties of Dynamical Systems by Quasi-Compactness. Lecture Notes in Mathematics, vol. 1766. Springer, New York (2001)

[22] Ionescu-Tulcea, C.T., Marinescu, G.: Théorème ergodique pour des classes d'opérations non complètement continues. Ann. Math. 52(1), 140-147 (1950) 
[23] Kautzsch, J.: Renewal Theory for Operators in Banach Spaces. Diplomarbeit Universität Bremen (2011)

[24] Kautzsch, J., Kesseböhmer, M., Samuel, T., Stratmann, B.O.: On the asymptotics of the $\alpha$-Farey transfer operator. Nonlinearity 28, 143-166 (2015)

[25] Keller, G.: On the rate of convergence to equilibrium in one-dimensional systems. Commun. Math. Phys. 96, 181-193 (1984)

[26] Kesseböhmer, M., Kombrink, S.: Fractal curvature measures and Minkowski content for self-conformal subsets of the real line. Adv. Math. 230, 2474-2512 (2012)

[27] Kesseböhmer, M., Munday, S., Stratmann, B.O.: Strong renewal theorems and Lyapunov spectra for $\alpha$-Farey and $\alpha$-Lüroth systems. Ergod. Theory Dyn. Syst. 32(3), 989-1017 (2012)

[28] Kesseböhmer, M., Slassi, M.: A distribution limit law for the continued fraction digit sum. Math. Nachrichten 281(9), 1294-1306 (2008)

[29] Kesseböhmer, M., Stratmann, B.O.: A multifractal analysis for Stern-Brocot intervals, continued fractions and Diophantine growth rates. J. Reine Angew. Math. 605, 133-163 (2007)

[30] Kleban, P., Özlük, A.: A Farey fraction spin chain. Commun. Math. Phys. 203, 635-647 (1999)

[31] Knauf, A.: On a ferromagnetic spin chain. Commun. Math. Phys. 153, 77115 (1993)

[32] Knauf, A.: The number-theoretical spin chain and the Riemann zeros. Commun. Math. Phys. 196, 703-731 (1998)

[33] Lanford, O.E., Ruedin, L.: Statistical mechanical methods and continued fractions. Helv. Phys. Acta 69, 908-948 (1996)

[34] Mayer, D.H.: Continued fractions and related transformations. In: Bedford, T., Keane, M., Series, C. (eds.) Ergodic Theory, Symbolic Dynamics and Hyperbolic Spaces, Oxford University Press, Oxford (1991)

[35] Melbourne, I., Terhesiu, D.: Operator renewal theory and mixing rates for dynamical systems with infinite measure. Invent. Math. 189, 61-110 (2012)

[36] Pomeau, Y., Manneville, P.: Intermittency transition to turbulence in dissipative dynamical systems. Commun. Math. Phys. 74, 189-197 (1980)

[37] Rychlik, M.: Bounded variation and invariant measures. Stud. Math. LXXVI, 69-80 (1983)

[38] Sarig, O.: Subexponential decay of correlations. Invent. Math. 150, 629-653 (2002)

[39] Schuster, H.G.: Deterministic Chaos. VCH, New York (1988)

[40] Thaler, M.: The asymptotics of the Perron-Frobenius operator of a class of interval maps preserving infinite measure. Stud. Math. 143(2), 103-119 (2000)

[41] Tyran-Kaminska, M.: Weak convergence to Lévy stable processes in dynamical systems. Stoch. Dyn. 10(2), 263-289 (2010) 
Johannes Kautzsch, Marc Kesseböhmer and Tony Samuel Fachbereich 3 - Mathematik und Informatik

Universität Bremen

Bibliothekstr. 1

28359 Bremen, Germany

e-mail: tony@math.uni-bremen.de

Johannes Kautzsch

e-mail: kautzsch@math.uni-bremen.de

Marc Kesseböhmer

e-mail: mhk@math.uni-bremen.de

Tony Samuel

Mathematics Department

California Polytechnic State University

San Luis Obispo, CA 93407-0403, USA

Communicated by Dmitry Dolgopyat.

Received: October 30, 2014.

Accepted: September 8, 2015. 